E15-2004-132

\title{
EXPERIMENTAL INVESTIGATION
}

OF MUON-CATALYZED $d t$ FUSION IN WIDE RANGES OF $D / T$ MIXTURE CONDITIONS

Submitted to «ЖЭТФ» 
V.R.Bom ${ }^{1}$, A. M. Demin ${ }^{2}$, D.L. Demin ${ }^{3}$, C. W.E. van Eijk ${ }^{1}$, M.P. Faifman ${ }^{4}$, V. V. Filchenkov ${ }^{3}$, A. N. Golubkov ${ }^{2}$, N. N. Grafov ${ }^{3}$, S. K. Grishechkin ${ }^{2}$, K. I. Gritsaj ${ }^{3}$,

V. G. Klevtsov ${ }^{2}$, A. D. Konin ${ }^{3}$, A. V. Kuryakin², S. V. Medved ${ }^{3}$, R. K. Musyaev², V. V. Perevozchikov², A. I. Rudenko ${ }^{3}$,

S. M. Sadetsky ${ }^{5}$, Yu. I. Vinogradov ${ }^{2}$, A. A. Yukhimchuk ${ }^{2}$,

S. A. Yukhimchuk ${ }^{3}$, V. G. Zinov ${ }^{3}$, S. V. Zlatoustovskii ${ }^{2}$

${ }^{1}$ Delft University of Technology, 2629 JB Delft, the Netherlands

${ }^{2}$ Russian Federal Nuclear Center, All-Russian Research Institute of Experimental Physics (RFNC-VNIIEF), 607200 Sarov, Nizhny Novgorod Region, Russia

${ }^{3}$ Joint Institute for Nuclear Research, Dzhelepov Laboratory of Nuclear Problems, 141980 Dubna, Russia

${ }^{4}$ Russian Research Center «Kurchatov Institute», 123182 Moscow, Russia

${ }^{5}$ St. Petersburg Nuclear Physics Institute, 188350 Gatchina, Russia 
Бом В.Р. и др.

E15-2004-132

Экспериментальное исследование реакции

мюонного катализа $d t$ в широком диапазоне условий смеси $D / T$

На фазотроне ЛЯП (ОИЯИ) выполнена обширная программа по экспериментальному исследованию реакции мюонного катализа $d t$. Получены параметры цикла $d t$ в широком диапазоне условий смеси $D / T$ : температуры 20-800 K, плотности 0,2-1,2 LHD и концентрации трития 15-86\%. Обобщаются полученные результаты.

Работа выполнена в Лаборатории ядерных проблем им. В.П.Джелепова ОИЯИ.

Препринт Объединенного Института Ядерных Исследований. Дубна, 2004

Bom V.R. et al.

E15-2004-132

Experimental Investigation of Muon-Catalyzed $d t$ Fusion

in Wide Ranges of $D / T$ Mixture Conditions

A vast program of the experimental investigation of muon-catalyzed $d t$ fusion was performed at the JINR Phasotron. Parameters of the $d t$ cycle were obtained in a wide range of $D / T$ mixture conditions: temperatures of $20 \div 800 \mathrm{~K}$, densities of $0.2 \div 1.2 \mathrm{LHD}$ and tritium concentrations of $15 \div 86 \%$. The results obtained are summarized.

The investigation has been performed at the Dzhelepov Laboratory of Nuclear Problems, JINR. 


\section{INTRODUCTION}

Investigation of the muon-catalyzed fusion (MCF) process is a unique independent direction of the modern physics relevant for molecular, atomic and nuclear physics [1, 2, 3, 4, 5, 6], and for astrophysics [7]. Study of the nuclear fusion reactions from the bound states of a muonic molecule is of great importance for determination of properties of the lightest nuclei, including various exotic nuclear systems. In addition, the high neutron yield of MCF can be effectively used for solving different practical problems such as the construction of an intense 14-MeV neutron source [8] and a nuclear fuel breeder [9].

That is why the process of MCF in the hydrogen isotope mixtures has been under active study in many laboratories in the world during the last several decades. During this period many experimental results have been obtained in the investigation of the muon-induced processes in different mixtures of hydrogen, deuterium and tritium as well as in pure isotopes, and most of them are in good agreement with theory. The most impressive achievement is the precise agreement between experiment and theory in the temperature dependence of the $d d \mu$-molecule formation rate in gaseous deuterium $[4,10,11]$. This allowed the binding energy of the loosely bound state of $d d \mu$ to be determined with a very high accuracy, $\epsilon_{11}^{\exp }=-1962.56_{-47}^{+32} \mathrm{meV}[4,10]$, which should be compared with the theoretical value $\epsilon_{11}^{\text {theor }}=-1964.83 \mathrm{meV}$.

In comparison with pure deuterium, the MCF process in a $D / T$ mixture manifests much richer physical phenomena (muon transfer $d \rightarrow t$ from the ground and excited states of the $d \mu$-atom, epithermal and many-body effects in the $d t \mu$ molecule formation). Theory predicts a significant increase of the $d t \mu$-molecule formation rate on the $D_{2}$ and $D T$ molecule with the rise of temperature and density of the mixture $[12,13]$. So, for the complete theoretical analysis it is necessary to measure temperature and density dependencies of the $D / T$ cycle parameters in as large ranges as possible. Finally, the results of the experimental and theoretical study of MCF processes in a double $D / T$ mixture will be rather helpful for investigation and explanation of the most difficult case of the triple $H / D / T$ mixture.

Previously, the really systematic experimental study of the MCF process was performed at PSI only for a low-density $(\varphi \simeq 0.1 \mathrm{LHD}, \varphi$ is relative to the liquid hydrogen nuclei density LHD $=4.25 \cdot 10^{22} \mathrm{~cm}^{-3}$ ), low-temperature $(T<300 \mathrm{~K})$ gaseous $D / T$ mixture [14]. The same group, as well as the 
RIKEN-RAL team, made measurements with liquid and solid $D / T$ mixtures [15, 16]. The only group which investigated MCF in the high-density ( $\varphi \simeq 1$ LHD), high-temperature $(T \leq 600 \mathrm{~K})$ mixtures was the LAMPF team $[17,18]$. However, its measurements had a «prompt» character and caused many questions on the analysis. So, we decided to conduct a full set of measurements in a wide region of the experimental conditions spanning the density range $\varphi=(0.2-1.2)$ LHD and the temperature range $T=(20 \div 800) \mathrm{K}$.

The Dzhelepov Laboratory of Nuclear Problems made a prominent contribution to the MCF experimental study. The Dubna group discovered the phenomenon of the $d d \mu$-molecule resonance formation [19] and later directly confirmed its existence by measurements of the temperature dependence of the $d d \mu$-molecule formation rate [20]. This group was the first to experimentally investigate [21] the muon-catalyzed fusion

$$
d t \mu \longrightarrow{ }^{4} \mathrm{He}(3.5 \mathrm{MeV})+n(14.1 \mathrm{MeV})+\mu
$$

and to confirm the theoretical predictions [22] of the high intensity of this process which induced the activity in the MCF study in the world.

Since 1997 our collaboration has been carrying out a large program of investigation of MCF processes in $D / T$ at the JINR Phasotron. The distinctive characteristic of our study is the use of the novel methods both in the measurements and in the experimental data analysis, which allows us to obtain accurate and reliable data not worse than those obtained at the meson facilities. The experimental method used by us made it possible to measure the MCF cycle parameters in the $D / T$ mixture under a wide variety of mixture conditions [23].

This paper is the report on the most comprehensive measurements of the MCF parameters in the $D / T$ mixture. The preliminary data were published in $[23,24$, $25,26]$. Figure 1 shows the condition ranges of the experiments conducted up to now.

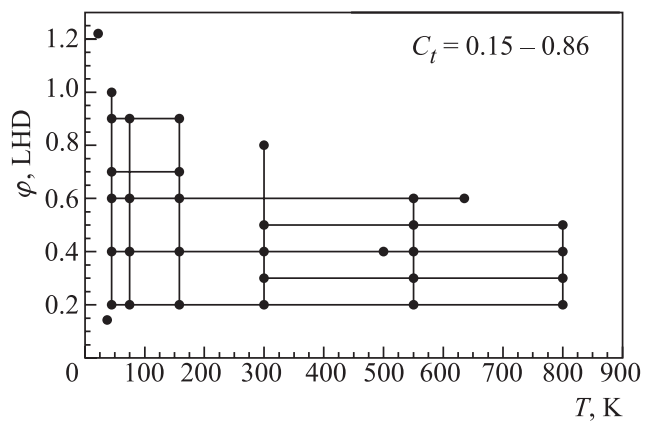

Fig. 1. The experimental conditions (density and temperature) for the MCF process study in the $D / T$ mixtures 
The accumulated data and the MCF cycle parameters cover wide ranges of $D / T$ mixture conditions:

- temperatures of $20 \div 800 \mathrm{~K}$,

- tritium concentrations of $15 \div 86 \%$,

- densities of $0.2 \div 1.2$ LHD.

\section{GENERAL DESCRIPTION OF THE PROCESS}

The simplified scheme of MCF kinetics in a double $D / T$ mixture is shown in Fig. 2. Muons stopped in the mixture form $d \mu$ and $t \mu$ atoms in their ground states with the probabilities $w_{d \mu}=C_{d} \cdot q_{1 S}$ and $w_{t \mu}=C_{t}+C_{d} \cdot\left(1-q_{1 S}\right)=1-C_{d} \cdot q_{1 S}$, respectively, where $C_{d}$ and $C_{t}$ are relative hydrogen isotope concentrations and $q_{1 S}$ is the fraction of $d \mu$ atoms in the ground state after muon cascade processes, which takes into account the muon transfer $(d \mu)_{n} \rightarrow(t \mu)_{n}$ from $d \mu$ to $t \mu$ during the deexcitation cascade $[27,28,29]$ :

$$
q_{1 S}=\frac{\lambda_{\mathrm{dex}}}{\lambda_{\operatorname{dex}}+\lambda_{\mathrm{tr}}} .
$$

Here $\lambda_{\operatorname{dex}}$ and $\lambda_{\text {tr }}$ are the rates of deexcitation and muon transfer averaged over the $d \mu$-atom exciting states. One should expect strong dependence of $q_{1 S}$ on $C_{t}$ and $\varphi[30,31]$.

The «standard» cascade model, in which initial $\mu$-atom energies are distributed around $E_{0}=(1-2) \mathrm{eV}$, is apparently valid only at very low densities $\varphi \leq 10^{-3}$ LHD. Now it is known that during the cascade muonic atoms can be both thermalized and accelerated getting the energy of even tens of eV (see, for example, $[32,33,34])$. However, till now the problem of knowing the initial energy distribution of muonic atoms after the cascade has not been solved definitely.

Being in the $d \mu$-atom ground state, the muon can be transferred to tritium in the collisional process

$$
d \mu+t \longrightarrow t \mu+d
$$

with the rate $\lambda_{d t}=2.8 \cdot 10^{8} \mathrm{~s}^{-1} \cdot \varphi[21,18,35,36]$. In the transfer process (2) the $t \mu$ atom acquires the energy $19 \mathrm{eV}$. Atoms $t \mu$ are formed in two hyperfine states with the total spin $F=1$ (weight 0.75 ) and $F=0$ (weight 0.25 ) and can take part in the spin-flip processes

$$
(t \mu)_{F=1}+t \longleftrightarrow(t \mu)_{F=0}+t .
$$

Muonic atoms $t \mu$ can form $d t \mu$ and $t t \mu$ molecules and $d \mu$ atoms can form $d d \mu$ molecules. The fusion reactions occur in these $\mu$ molecules in which the muon can be released and stimulate the next MCF cycle or stick to helium produced 


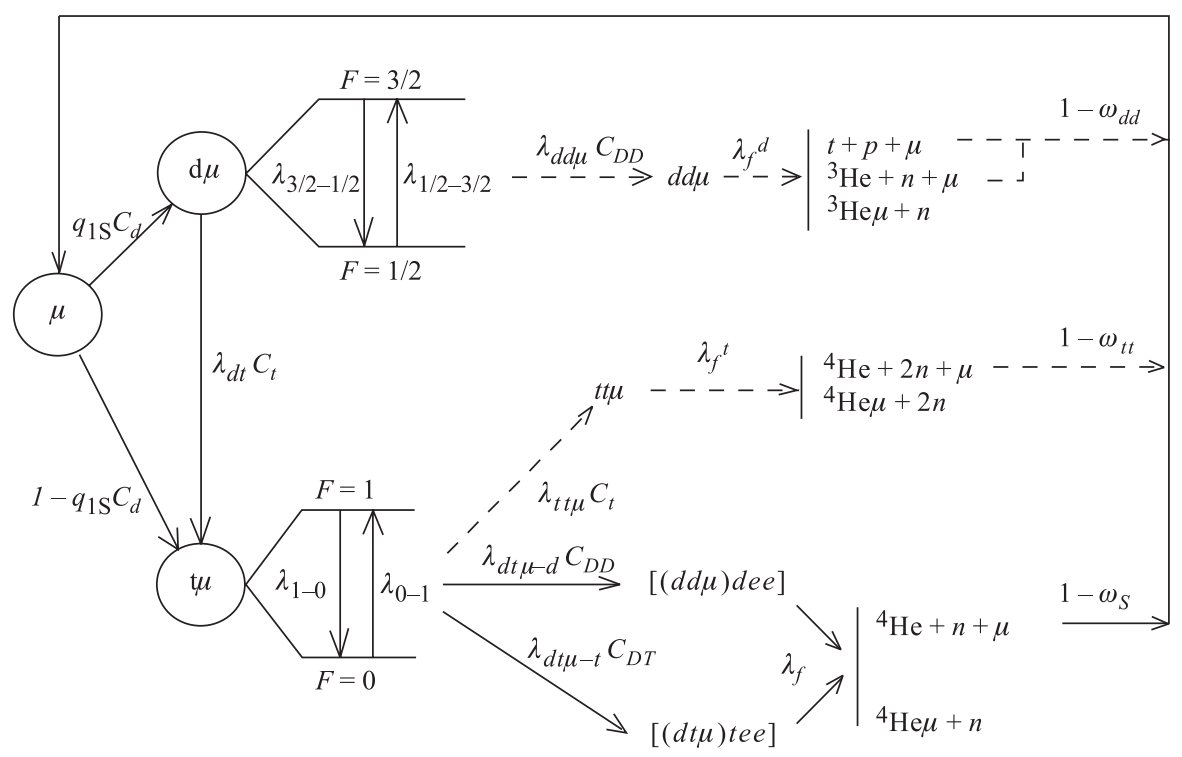

Fig. 2. Scheme of MCF kinetics in the double $D / T$ mixture

in the reactions. The appropriate notations for the rates of muonic formation and fusion reactions as well as for the sticking probabilities are indicated in Fig. 2. Being bound in the $\mu$ atom or $\mu$ molecule or being free, the muon disappears with the rate $\lambda_{0}=4.55 \cdot 10^{5} \mathrm{~s}^{-1}$.

The specific feature of the $d d \mu$ - and $d t \mu$-molecule formation processes is their resonance character, that is, the muonic molecular formation rates $\lambda_{d d \mu}$ and $\lambda_{d t \mu}$ turn out to depend on the $\mu$-atom kinetic energy [3]. The MCF $d+d$ cycle has been studied very well. The measured temperature dependence $\lambda_{d d \mu}(T)$ is in excellent agreement with theory [13].

Quite a different situation takes place for the MCF $d+t$ cycle. Really, this process has been studied in detail in that parameter region (low temperatures) where the «standard» theory predicts its relatively low intensity. It follows from experiment that just in this region the MCF process turns out to be very effective. Modern theory explains this only qualitatively.

It follows from the original Vesman consideration [37] that the resonance $d t \mu$-molecule formation occurs in the interaction of the $t \mu$ atom with $D_{2}, D T$ or $H D$ molecules according to the scheme [22]:

$$
t \mu+(D X)_{K_{i}} \longrightarrow[(d t \mu), x, 2 e]_{\nu, K_{f}} \quad X=H, D, T \quad x=p, d, t,
$$

where the energy released under $d t \mu$ formation together with the $t \mu$-atom kinetic energy $E_{t \mu}$ is transferred to excite the vibration-rotational state of the molecular 
complex $[(d t \mu), x, 2 e]$. Here $K_{i}$ and $K_{f}$ are the rotational quantum numbers of the «initial» molecule $D X$ and the «final» complex respectively. The set of the resonance $t \mu$ atom energies

$$
E_{t \mu}^{r}=\Delta E_{\nu, K}
$$

corresponds to different transitions $\nu=3,4,5 ; K_{i} \rightarrow K_{f}$. Really, the spin states of the $t \mu$ atom and the $d t \mu$ molecule should be taken into account for determination of $E_{t \mu}^{r}$. In addition, the position and intensity of the resonances depend on the type of molecule $\left(D_{2}, D T\right.$ and $\left.H D\right)$ and the temperature of the mixture influencing the population of the rotational states of these molecules.

Being formed, the complex $[(d t \mu), x, 2 e]$ either undergoes the back decay $[(d t \mu), x, 2 e] \rightarrow t \mu+D X$ or the fusion reactions

$$
\begin{gathered}
d t \mu \longrightarrow{ }^{4} \mathrm{He}+n+\mu, \\
d t \mu \longrightarrow{ }^{4} \mathrm{He} \mu+n
\end{gathered}
$$

take place in it (with the high rate $\lambda_{f} \simeq 10^{12} \mathrm{~s}^{-1}$ [38]. The muon-to-helium sticking probability is $\omega_{s} \simeq 0.5 \%$.

The resonance dependencies $\lambda_{d t \mu-p, d, t}\left(E_{t \mu}\right)$ for the $t \mu$ atom of spin $F=0$ are shown in Fig. 3, where presented calculations are based on the evaluation scheme developed in [13].

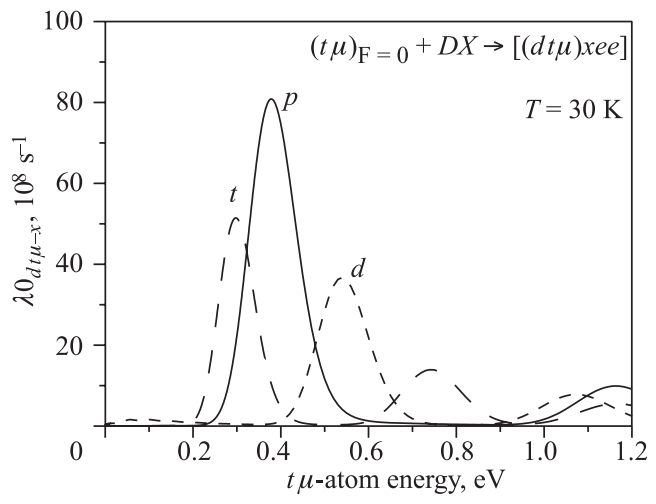

Fig. 3. The $d t \mu$-molecule formation rates on $D_{2}, D T$ and $H D$ molecules for the $t \mu$-atom spin $F=0$ as a function of $E_{t \mu}$ for $T=30 \mathrm{~K}$ (calculations based on [13])

The following remarkable features are evident from this figure:

1. Resonance formation of the $d t \mu$ molecule on $H D$ molecules is the most intensive.

2. The resonance positions correspond to relatively high $t \mu$-atom energies, that is, to high temperatures $\left(T \sim 10^{3} \mathrm{~K}\right)$ for the thermalized muonic atoms. 
3. The positions of the resonances of each type corresponding to various vibration levels of the complex $\nu=3,4,5$. The nearest resonance for $\lambda_{d t \mu-d}^{0}$ is placed at $E_{t \mu} \simeq 0.5 \mathrm{eV}$. It means that the nearest «sub-threshold» resonance (corresponding to $\nu=2$ ) lies close to zero at the negative $t \mu$-atom energy $E_{t \mu} \simeq-(10-12) \mathrm{meV}$. Negative energy means that for the most intensive dipole transitions $\left|K_{f}-K_{i}\right|=1$ the energy excess arises which cannot be transferred in two-particle reaction (4).

For the highest multiple transitions $\left(\nu=2 ; K_{i}=0,1 \rightarrow K_{f}=2,3,4\right)$ process (4) becomes possible but its intensity is two orders of magnitude lower than that of the main transitions. However, in contradiction with the «standard» theory, experiment manifests high MCF intensity in the low temperature $D / T$ mixture and reveals non-trivial density dependence of its cycling rate. Note that the highest value of the cycling rate $\Lambda_{c}=(185 \pm 13) \mu \mathrm{s}^{-1}$ was measured at PSI [15] in a solid $D / T$ mixture.

This can be qualitatively explained in the modern theory [39], according to which influence of the sub-threshold resonance turns out to be much stronger due to the mechanism of triple collisions. According to the theory, the resonance $d t \mu$ formation at low temperatures occurs at sub-threshold resonance in the triple collision process

$$
t \mu+D_{2}+M \longrightarrow[(d t \mu), d, 2 e]+M^{\prime}, \quad M=D_{2}, D T, T_{2} .
$$

The «additional» second molecule $M$ plays the role of a spectator which carries away the energy excess. As (7) is a three-particle process, it must depend on the density of molecules $M$.

Qualitatively scheme (7) explains both the high values of $\lambda_{d t \mu-d}$ and its density dependence observed in experiment. However, in spite of many efforts undertaken to calculate its intensity (see, for example, [40, 41]), the quantitative explanation is not obtained yet.

With the temperature increase, the resonance pictures are modified due to the change of the population of the $D X$ molecule rotational states and the thermal motion of the molecule. The calculated rates $\lambda_{d t \mu-d}$ and $\lambda_{d t \mu-t}$ as functions of $E_{t \mu}$ for $T=300 \mathrm{~K}$ are presented in Fig. 4 .

The Maxwell distribution for the thermalized $t \mu$ atoms is shown in one of them $\left(\lambda_{d t \mu-t}\right)$. As is seen, this distribution only slightly overlaps the nearest resonance.

The resonances for $\lambda_{d t \mu}$ at $T=1000 \mathrm{~K}$ are presented in Fig. 5. In this case the Maxwell distribution considerably overlaps the most intensive resonances for $d t \mu$ formation on $D_{2}, D T$ and $H D$ molecules. Unfortunately, this high temperature is not yet achieved in experiment. The temperature $T=800 \mathrm{~K}$ is the highest at which the measurements were made (in Dubna).

As we mentioned, a substantial part of $t \mu$ has an initial (after cascade) energy $E_{t \mu}>1 \mathrm{eV}$. In elastic collisions $t \mu+t, t \mu+d$ these atoms are quickly 

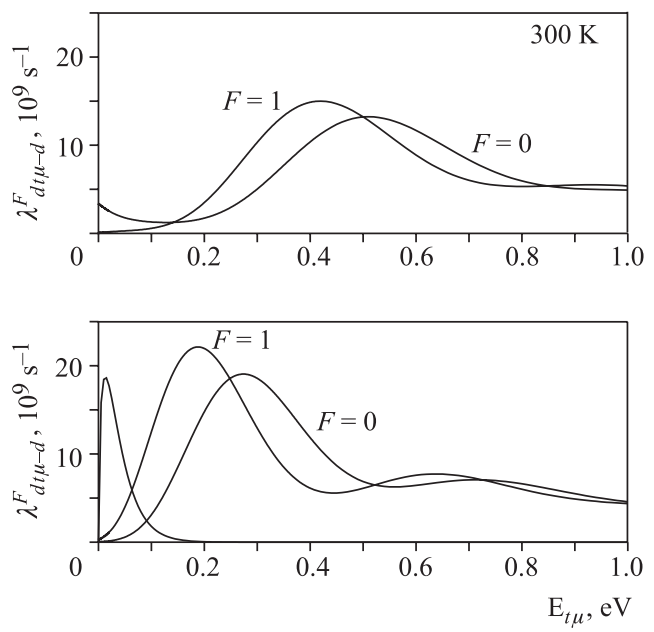

Fig. 4. The dependence $\lambda_{d t \mu-d}$ (top) and $\lambda_{d t \mu-t}$ (bottom) on the $t \mu$-atom energy for $T=300 \mathrm{~K}$. Maxwell distribution is shown in the bottom picture

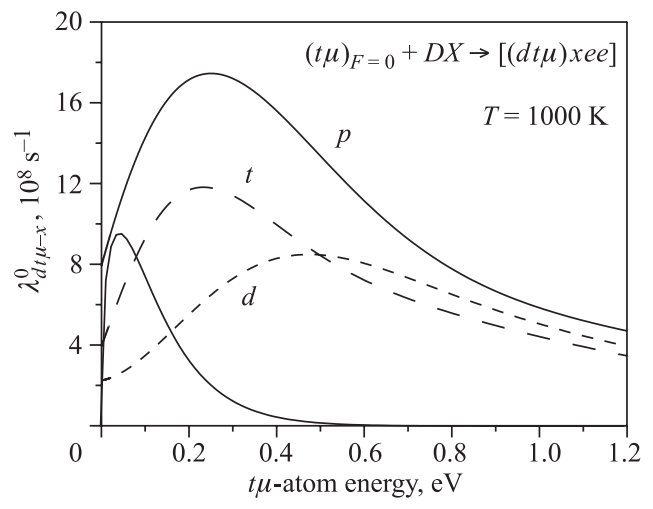

Fig. 5. The $d t \mu$-molecule formation rates on $D_{2}, D T$ and $H D$ molecules for the $t \mu$-atom spin $F=0$ as a function of $E_{t \mu}$ for $T=1000 \mathrm{~K}$ (calculations based on [13]) and Maxwell distribution

thermalized. The thermalization time is $\sim$ ns for the 1 LHD of a mixture. Accordingly, the time distribution of the fusion reaction products (neutrons) should have two components: the quick «spike» corresponding to the first pass through the resonances and the much slower «steady-state» component.

Due to shortness of the epithermal spike and ambiguity in the $t \mu$-atom initial energy it is hard to interpret this effect. That is why the main efforts of different experimental groups were concentrated on the steady-state study for which the $t \mu$-atom energy spectrum is a Maxwell distribution. 
For convenience the comparison of the measurements with the theoretical calculations are performed for the so-called «effective» $d t \mu$-molecule formation rate as a function of temperature. This is obtained by integrating over all possible initial states, averaging over all final states and convolving with the Maxwell spectrum $W\left(E_{t \mu}, T\right)$. Such calculations for the $D / T$ mixture were made in [13].

For the steady-state the time distribution of fusion neutrons has the form

$$
d N_{n} / d t=N_{\mu} \cdot \epsilon_{n} \cdot \Lambda_{c} \cdot \exp \left(-\lambda_{n} t\right), \quad \lambda_{n}=\lambda_{0}+w \Lambda_{c} .
$$

Here $\Lambda_{c}=\lambda_{c} \varphi ; \epsilon_{n}$ is the neutron detection efficiency; $N_{\mu}$ is the number of muons stopped in the $D / T$ mixture; $\omega$ is the muon loss in the cycle which is the probability of muon sticking to helium in fusion reactions, mainly in $d+t$ $\left(\omega_{s}\right)$ and also, with lower weight, in the accompanied reactions $d+d$ and $t+t$. The cycling rate $\Lambda_{c}$ means the inverse of the averaged time between the closest cycles. It involved mainly the time of $d \mu-t \mu$ transfer (2), $t \mu$-atom spin-flip process (3) and $d t \mu$-molecule formation (4). The neutron yield $Y_{n}$ is limited by $\omega$ and $\lambda_{0}$ :

$$
Y_{n}^{-1}=\omega+\frac{\lambda_{0}}{\Lambda_{c}} .
$$

The expression for $\lambda_{c}$, corresponding to the kinetic scheme of Fig. 2, has the form

$$
\frac{1}{\lambda_{c}}=\frac{q_{1 s} \cdot C_{d}}{\lambda_{d t} \cdot C_{t}}+\frac{0.75}{\lambda_{1-0} C_{t}}+\frac{1}{\lambda_{d t \mu-d} \cdot C_{D D}+\lambda_{d t \mu-t} \cdot C_{D T}} .
$$

To extract the values $\lambda_{d t \mu-d}$ and $\lambda_{d t \mu-t}$ one should analyze, using formula (10), the experimental values of $\lambda_{c}$ measured at different tritium concentrations changing the relative population of $D_{2}$ and $D T$ molecules.

The expression for $\omega$ has the form

$$
\begin{aligned}
& \omega=\omega_{s}+\frac{\lambda_{t t \mu} C_{t} \cdot \omega_{t t}}{\lambda_{d t \mu-d} C_{D D}+\lambda_{d t \mu-t} C_{D T}+\lambda_{t t \mu} C_{t}}+ \\
& \frac{q_{1 s} C_{d} \cdot \frac{2}{3} \cdot \lambda_{d d \mu}^{3 / 2} C_{D D} \cdot \omega_{d d} \cdot r}{\lambda_{d t} C_{t}+\lambda_{d d \mu}^{3 / 2} C_{D D}+\lambda_{3 / 2-1 / 2} C_{d}}+\frac{\lambda_{Z} C_{Z}}{\lambda_{c}} .
\end{aligned}
$$

Here $\lambda_{Z}$ is the rate of muon transfer to possible admixtures with $Z>1$, having concentration $C_{Z} ; \lambda_{d d \mu}^{3 / 2}$ is the rate of $d d \mu$-molecule formation from the $d \mu$-atom state with spin $F=3 / 2 ; r$ is the branching ratio of the $d d$ fusion channels $\left({ }^{3} \mathrm{He}+n\right)$ and $(t+p)$; others are seen in Fig. 2. It follows from Eq. (11), that minimum value of $\omega$ is achieved at highest $\lambda_{c}$ (large $\lambda_{d t}$ and $\lambda_{d t \mu-d, t}$ ) where $\omega$ is close to its natural limit $\omega_{s} \simeq 0.5 \%$.

Note that in expressions (10), (11) and in what follows the cycling rate as well as all collisional rates are normalized to the nuclear density $\varphi$ of the $D / T$ mixture. 


\section{EXPERIMENTAL METHOD}

All experimental runs were made at the installation «Triton» mounted on the muon channel [42] of the JINR Phasotron. The experimental setup is schematically shown in Fig. 6.
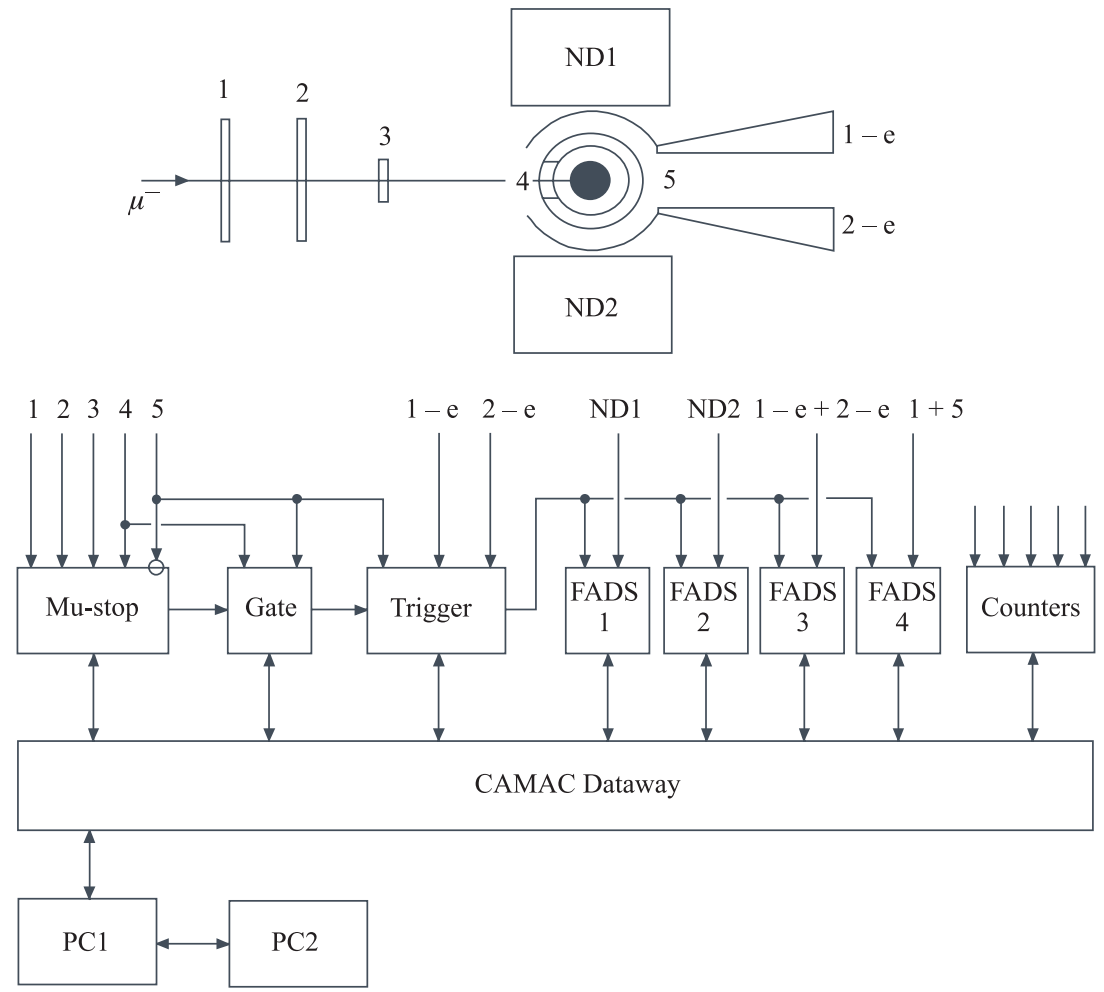

Fig. 6. Experimental layout

The novel experimental method was used [23]. Based on measurements of the total charge produced by the fusion neutrons in a detector, it allowed us to avoid the distortions in the neutron time spectra caused by the pile-up and thus to use a high-efficiency detection system.

Incoming muons are detected by scintillation counters 1, 2, 3, a proportional wire counter 4 and stopped in the target. Neutrons from the $d-t$ reaction are detected by two full-absorption neutron detectors ND1, ND2. Electrons from the decay of muons stopped in the target are registered by the proportional wire counter 5 and scintillation detectors 1-e, 2-e. 
2.1. The Specific Features of the Method. The following important features characterize the method used.

1. Unique targets and tritium handling system were used, which allowed measurements in a wide range of the $D / T$ mixture densities and temperatures.

2. A high-efficiency neutron detection system was used in the geometry close to $4 \pi$. It provided a high counting rate and low accidental background.

3. A specially designed proportional counter was used for muon and electron detection; having a low sensitivity to neutrons, it allowed reliable electron identification.

4. Time distributions of charge were measured instead of usually registered time spectra of the number of events. Flash ADC were used for this aim. It allowed us to avoid distortions in the neutron time spectra and thus to use a high-efficiency detection system.

5. The novel analysis methods were used, which turned out to be most effective for the high neutron multiplicity realized in the experiment. In addition to the usually measured neutron time distribution we measured and analyzed the neutron multiplicity distribution and the spectra of the time between the $\mu$-decay electron and the last neutron in the series. This allowed us to decrease the systematic errors and to obtain reliable data.

2.2. Targets and Gas Handling System. A set of targets [43, 44, 45] with the working volume of $10-18 \mathrm{~cm}^{3}$ depending on the tritium content was used in the experiments. The targets allowed the following measurements:

- with liquid $D / T$ (the liquid tritium target (LTT) [43] of $18 \mathrm{~cm}^{3}$, working temperature $20-40 \mathrm{~K}$, pressure up to $20 \mathrm{bar}$ );

- with hot gaseous $D / T$ (the high pressure tritium target (HPTT) [44] of $16 \mathrm{~cm}^{3}$, working temperature $300-800 \mathrm{~K}$, pressure up to 1600 bar);

- with cold gaseous $D / T$ (two high pressure tritium targets (HPTT) [45] of 8 and $16 \mathrm{~cm}^{3}$, working temperature $40-200 \mathrm{~K}$, pressure up to $2500 \mathrm{bar}$ ).

The special cryogenic system [43] (for the LTT and HPTT) and the cryorefrigerator (for the HPTT) were used at low temperatures $(T<300 \mathrm{~K})$ and the system of special hitters was used at high temperatures to maintain the needed temperature regime. Cryogenic filling is used for all targets.

A special preparation system based on palladium filters [46] provided the gas of required composition and purified of impurities at the level $<10^{-7}$ of volume parts. The molecular composition of the mixtures was monitored with the aid of chromatography.

2.3. Detectors and Electronics. The target was surrounded by a set of detectors. Scintillation counters 1-3 detected incoming muons. A cylinder-shaped proportional counter (PC, analogous to [47] with wires grouped in two parts $(4,5)$ served to select muon stops in the target (signal $1 \cdot 2 \cdot 3 \cdot 4 \cdot \overline{5}$ ) and to detect electrons from the muon decay. Specially designed cylinder-shaped scintillation counters (SC) 1-e, 2-e were used to detect $\mu$-decay electrons in coincidence with counter 

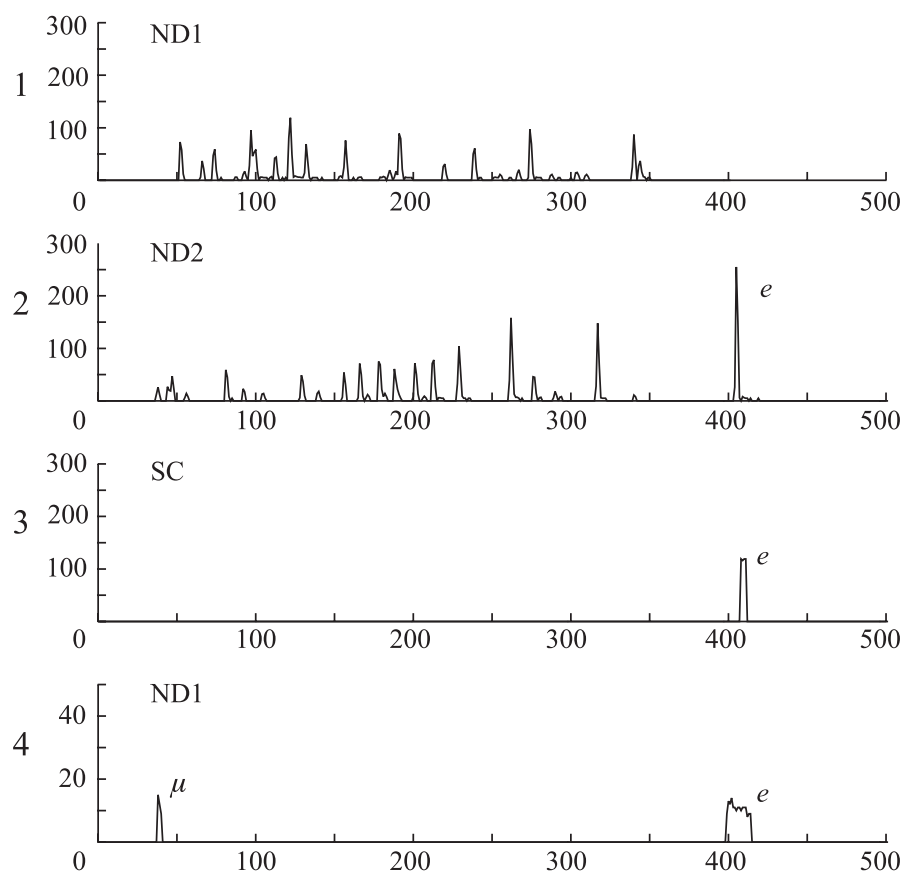

Fig. 7. Flash ADC signals for a single muon

5 (signals $5 \cdot 1$-e and $5 \cdot 2$-e were considered as a $\mu$-decay electron). The fullabsorption neutron spectrometer [48, 49] consisting of two large detectors (ND1 and ND2) with a volume of $12.5 \mathrm{l}$ each was the basis of the detection system. It was aimed at detecting neutrons from reactions (5), (6). Plastic scintillator with dimensions $(\oslash 31 \times 17 \mathrm{~cm})$ was used in each detector. It was viewed by four PMs XP 2040. The direct contact of the PMs with the scintillator and teflon used as an optical reflector provided excellent spectrometric properties of the detector. Its energy resolution was $\sigma_{F W H M}=0.09 \cdot\left(1+1 / \sqrt{E_{e}, \mathrm{MeV}}\right)$. The total solid angle covered by two detectors was $\Omega \simeq 70 \%$, which corresponded to the total neutron detection efficiency $\epsilon_{n} \simeq 2 \cdot 15 \%$. The time resolution of ND was dictated by the light collection process and electronics and was $\Delta t=6-7 \mathrm{~ns}$.

The trigger [50] allows recording of only those events for registration which were connected with an electron detection. As the intensity of the process under study was high, these events were accompanied by neutron detection practically in each case.

The trigger requirements included the presence of the muon stop signals $(1,2,3,4, \overline{5})$ and electron signals $(5,1-e$ or $5,2-e)$ during the time gate $20 \mu \mathrm{s}$ long started by the incoming muon signal $(1,2)$. Insertion of the electron signal 
in the trigger makes it possible to radically suppress the background connected with the muon stops in the target walls, where muon undergoes predominately (90\%) nuclear capture without electron escape. Additional suppression of this background is achieved under the condition when only delayed electrons (later than $0.2 \mu$ s after the gate start) are permitted.

Another important advantage of this is that the direct normalization to the electron number becomes possible without a necessity to determine the number of muon stops in hydrogen. This method was first employed by us in the first experiment on the MCF $d+t$ reaction [21] and allows successful accomplishment of this fundamental work.

Pulses from the neutron spectrometer are registered by the flash ADC $(8$ bits $\times 2048$ samples, $100 \mathrm{Mc} / \mathrm{s}$ ) producing a time distribution of the ND1, ND2 signal amplitude for each single muon. To provide correct time measurements, the signals of the detector for incoming muons and the electron counter are also analyzed by the flash ADC. An example of «oscillogramms» observed at the flash ADC is shown in Fig. 7. During each run the on-line monitoring of data accumulation was conducted.

\section{EXPERIMENTAL CONDITIONS}

A total of 81 exposures with $D / T$ mixtures were carried out. The conditions (density, temperature and tritium concentration) of each run are presented in Table 1. In each exposure (duration of 6-10 h) at least 20000 electrons from the decay of muons stopped in the target were accumulated. In practically all cases the neutron statistics was sufficiently large. The special exposures with empty targets were carried out to measure background of electrons from muons stopped in the target walls.

Table 1: Normalized cycling rates $\lambda_{c}$, muon loss probabilities $\omega$ and neutron yields per muon $Y_{n}$. For $\lambda_{c}$ the uncertainties due to statistics, density $(\Delta \varphi)$, charge calibration procedure $(\Delta q)$ and the total uncertainty are indicated. For all parameters the total errors include the systematic uncertainty in the determination of neutron detection efficiency $\left(\Delta \epsilon_{n}=6 \%\right.$ )

\begin{tabular}{|c|c|c|c|c|c|c|c|c|c|}
\hline \multirow[b]{2}{*}{ No. } & \multicolumn{2}{|c|}{ Conditions } & \multirow[b]{2}{*}{$\begin{array}{l}\omega, \\
\%\end{array}$} & \multirow[b]{2}{*}{$Y_{n}$} & \multirow[b]{2}{*}{$\begin{array}{c}\lambda_{c} \\
\mu \mathrm{s}^{-1}\end{array}$} & \multicolumn{4}{|c|}{ Error contributions (\%) } \\
\hline & $\begin{array}{c}\varphi, \\
\text { LHD }\end{array}$ & $\begin{array}{c}C_{t}, \\
\%\end{array}$ & & & & Stat. & $\Delta \varphi$ & $\Delta q$ & Total \\
\hline \multicolumn{10}{|c|}{$T=(22.0 \pm 0.7) \mathrm{K}$ (liquid) } \\
\hline 1 & 1.19 & $18.1(1.5)$ & $0.77(0.07)$ & $68.9(5.9)$ & 61.5 & 0.4 & 2.1 & 3.1 & 7.7 \\
\hline 2 & 1.20 & $33.4(1.0)$ & $0.72(0.06)$ & $96.6(7.9)$ & 117.7 & 0.2 & 2.1 & 3.1 & 7.7 \\
\hline 3 & 1.19 & $35.2(1.0)$ & $0.63(0.05)$ & $102.9(8.6)$ & 117.9 & 0.3 & 2.1 & 3.1 & 7.7 \\
\hline 4 & 1.23 & $63.5(2.0)$ & $0.76(0.07)$ & $82.5(6.9)$ & $(84.6)$ & 0.4 & 2.4 & 3.1 & 7.8 \\
\hline
\end{tabular}


Continuation of Table 1

\begin{tabular}{|c|c|c|c|c|c|c|c|c|c|}
\hline \multirow[b]{2}{*}{ No. } & \multicolumn{2}{|c|}{ Conditions } & \multirow[b]{2}{*}{$\begin{array}{l}\omega, \\
\%\end{array}$} & \multirow[b]{2}{*}{$Y_{n}$} & \multirow[b]{2}{*}{$\begin{array}{c}\lambda_{c} \\
\mu \mathrm{s}^{-1}\end{array}$} & \multicolumn{4}{|c|}{ Error contributions (\%) } \\
\hline & $\begin{array}{c}\varphi, \\
\text { LHD }\end{array}$ & $\begin{array}{c}C_{t}, \\
\%\end{array}$ & & & & Stat. & $\Delta \varphi$ & $\Delta q$ & Total \\
\hline 5 & 1.24 & $85.5(2.5)$ & $1.40(0.11)$ & $34.2(2.8)$ & 20.8 & 0.6 & 2.4 & 3.1 & 7.8 \\
\hline \multicolumn{10}{|c|}{$T=(38.5 \pm 2.0) \mathrm{K}$} \\
\hline 6 & 0.143 & $57(2)$ & $1.03(0.08)$ & $10.1(0.8)$ & 31.1 & 1.5 & 3.3 & 3.1 & 8.2 \\
\hline \multicolumn{10}{|c|}{$T=(45 \pm 2) \mathrm{K}$} \\
\hline 7 & 0.237 & $31.4(0.5)$ & $0.77(0.06)$ & $32.0(2.6)$ & 80.5 & 1.0 & 3.4 & 3.4 & 8.3 \\
\hline 8 & 0.449 & $16.7(0.5)$ & $0.81(0.06)$ & $39.4(3.2)$ & 59.9 & 1.0 & 3.0 & 3.2 & 8.1 \\
\hline 9 & 0.450 & $31.4(0.5)$ & $0.68(0.05)$ & $54.2(4.5)$ & 88.7 & 0.9 & 3.3 & 3.4 & 8.3 \\
\hline 10 & 0.448 & $50.6(0.5)$ & $0.81(0.06)$ & $43.8(3.6)$ & 73.1 & 1.3 & 3.0 & 3.2 & 8.1 \\
\hline 11 & 0.445 & $71.1(0.5)$ & $1.46(0.11)$ & $19.0(1.6)$ & 30.6 & 1.3 & 2.9 & 3.2 & 8.1 \\
\hline 12 & 0.689 & $16.3(0.5)$ & $1.12(0.09)$ & $46.1(3.7)$ & 64.2 & 0.9 & 3.0 & 3.1 & 8.0 \\
\hline 13 & 0.643 & $31.1(0.5)$ & $0.74(0.06)$ & $69.9(5.8)$ & 101.2 & 0.7 & 3.3 & 3.4 & 8.2 \\
\hline 14 & 0.704 & $52.7(0.5)$ & $1.11(0.09)$ & $53.4(4.2)$ & 84.0 & 0.7 & 3.0 & 3.1 & 8.0 \\
\hline 15 & 0.766 & $71.2(0.5)$ & $1.89(0.15)$ & $27.5(2.2)$ & 34.7 & 1.3 & 3.0 & 3.1 & 8.1 \\
\hline 16 & 1.022 & $16.3(0.5)$ & $1.55(0.12)$ & $45.0(3.5)$ & 65.2 & 1.0 & 3.0 & 3.1 & 8.0 \\
\hline 17 & 0.912 & $31.1(0.5)$ & $0.89(0.07)$ & $76.0(6.3)$ & 118.5 & 0.8 & 3.2 & 3.4 & 8.2 \\
\hline 18 & 1.024 & $52.7(0.5)$ & $1.12(0.09)$ & $64.2(5.0)$ & 97.7 & 0.8 & 3.0 & 3.1 & 8.0 \\
\hline 19 & 1.018 & $71.2(0.5)$ & $1.83(0.14)$ & $34.2(2.7)$ & 40.6 & 1.1 & 3.0 & 3.1 & 8.1 \\
\hline \multicolumn{10}{|c|}{$T=(75 \pm 2) \mathrm{K}$} \\
\hline 20 & 0.234 & $31.4(0.5)$ & $0.85(0.07)$ & $29.8(2.5)$ & 81.2 & 1.0 & 3.0 & 3.4 & 8.2 \\
\hline 21 & 0.445 & $31.4(0.5)$ & $0.87(0.07)$ & $50.4(4.2)$ & 92.1 & 0.9 & 2.9 & 3.4 & 8.1 \\
\hline 22 & 0.635 & $31.4(0.5)$ & $0.94(0.07)$ & $69.9(5.8)$ & 101.6 & 0.7 & 3.0 & 3.4 & 8.1 \\
\hline 23 & 0.897 & $31.1(0.5)$ & $0.91(0.07)$ & $75.5(6.2)$ & 119.5 & 0.8 & 3.0 & 3.4 & 8.1 \\
\hline \multicolumn{10}{|c|}{$T=(158 \pm 2) \mathrm{K}$} \\
\hline 24 & 0.230 & $31.4(0.5)$ & $0.94(0.07)$ & $28.6(2.4)$ & 79.3 & 1.1 & 3.0 & 3.4 & 8.2 \\
\hline 25 & 0.438 & $16.7(0.5)$ & $1.41(0.11)$ & $31.0(2.5)$ & 58.7 & 1.1 & 3.0 & 3.2 & 8.1 \\
\hline 26 & 0.424 & $31.0(0.5)$ & $0.99(0.08)$ & $45.4(3.7)$ & 88.8 & 1.0 & 3.1 & 3.2 & 8.1 \\
\hline 27 & 0.436 & $31.4(0.5)$ & $0.88(0.07)$ & $48.1(4.0)$ & 90.9 & 0.9 & 3.0 & 3.4 & 8.1 \\
\hline 28 & 0.433 & $50.6(0.5)$ & $1.00(0.08)$ & $39.2(3.2)$ & 74.5 & 1.3 & 3.0 & 3.2 & 8.1 \\
\hline 29 & 0.430 & $71.1(0.5)$ & $2.01(0.15)$ & $17.3(1.4)$ & 29.5 & 1.3 & 3.0 & 3.2 & 8.1 \\
\hline 30 & 0.607 & $16.3(0.5)$ & $1.94(0.15)$ & $31.7(2.5)$ & 63.2 & 1.4 & 3.0 & 3.1 & 8.1 \\
\hline 31 & 0.620 & $31.1(0.5)$ & $0.98(0.08)$ & $57.9(4.8)$ & 100.1 & 0.9 & 3.1 & 3.4 & 8.2 \\
\hline 32 & 0.621 & $52.7(0.5)$ & $1.14(0.09)$ & $48.9(3.9)$ & 82.9 & 0.8 & 3.1 & 3.1 & 8.1 \\
\hline 33 & 0.688 & $71.2(0.5)$ & $1.64(0.13)$ & $27.1(2.1)$ & 35.4 & 1.3 & 3.1 & 3.1 & 8.1 \\
\hline 34 & 0.905 & $16.3(0.5)$ & $1.89(0.15)$ & $36.9(2.9)$ & 64.7 & 1.3 & 3.0 & 3.1 & 8.1 \\
\hline 35 & 0.876 & $31.1(0.5)$ & $0.90(0.07)$ & $72.4(6.0)$ & 119.6 & 0.8 & 3.0 & 3.4 & 8.1 \\
\hline 36 & 0.907 & $52.7(0.5)$ & $1.09(0.08)$ & $66.3(5.2)$ & 101.7 & 0.8 & 3.0 & 3.1 & 8.0 \\
\hline 37 & 0.902 & $71.2(0.5)$ & $1.62(0.12)$ & $34.9(2.8)$ & 40.6 & 1.1 & 3.0 & 3.1 & 8.1 \\
\hline \multicolumn{10}{|c|}{$T=(300 \pm 3) \mathrm{K}$} \\
\hline 38 & 0.204 & $31.4(0.5)$ & $1.23(0.10)$ & $28.2(2.3)$ & 91.4 & 1.7 & 3.4 & 3.5 & 8.5 \\
\hline 39 & 0.303 & $17.9(0.5)$ & $2.13(0.16)$ & $21.6(1.7)$ & 67.2 & 1.1 & 3.3 & 3.1 & 8.1 \\
\hline 40 & 0.302 & $36.1(0.5)$ & $1.14(0.09)$ & $36.7(3.0)$ & 101.1 & 0.9 & 3.4 & 3.1 & 8.2 \\
\hline
\end{tabular}


Continuation of Table 1

\begin{tabular}{|c|c|c|c|c|c|c|c|c|c|}
\hline \multirow[b]{2}{*}{ No. } & \multicolumn{2}{|c|}{ Conditions } & \multirow[b]{2}{*}{$\begin{array}{l}\omega, \\
\%\end{array}$} & \multirow[b]{2}{*}{$Y_{n}$} & \multirow[b]{2}{*}{$\begin{array}{c}\lambda_{c} \\
\mu \mathrm{s}^{-1}\end{array}$} & \multicolumn{4}{|c|}{ Error contributions (\%) } \\
\hline & $\begin{array}{c}\varphi, \\
\mathrm{LHD}\end{array}$ & $\begin{array}{c}C_{t}, \\
\%\end{array}$ & & & & Stat. & $\Delta \varphi$ & $\Lambda \Omega$ & Total \\
\hline 41 & 0.312 & $52.0(0.5)$ & $1.27(0.10)$ & $30.3(2.5)$ & 78.7 & 1.0 & 3.2 & 3.1 & 8.1 \\
\hline 42 & 0.312 & $68.8(0.5)$ & $1.25(0.10)$ & $21.4(1.7)$ & 47.6 & 1.0 & 3.2 & 3.1 & 8.1 \\
\hline 43 & 0.434 & $15.4(0.5)$ & $0.97(0.07)$ & $35.4(2.9)$ & 59.3 & 0.9 & 3.2 & 3.1 & 8.1 \\
\hline 44 & 0.411 & $31.0(0.5)$ & $1.08(0.08)$ & $43.7(3.6)$ & 96.0 & 1.0 & 3.2 & 3.2 & 8.1 \\
\hline 45 & 0.425 & $32.7(0.5)$ & $0.95(0.07)$ & $49.4(4.0)$ & 99.9 & 0.7 & 3.3 & 3.1 & 8.1 \\
\hline 46 & 0.443 & $35.0(1.0)$ & $0.89(0.07)$ & $53.6(4.4)$ & 104.4 & 0.5 & 3.4 & 3.2 & 8.2 \\
\hline 47 & 0.409 & $47.7(0.8)$ & $0.97(0.07)$ & $44.0(3.6)$ & 89.3 & 0.7 & 3.4 & 3.1 & 8.1 \\
\hline 48 & 0.411 & $68.5(0.5)$ & $1.21(0.09)$ & $27.7(2.2)$ & 50.3 & 0.8 & 3.2 & 3.1 & 8.1 \\
\hline 49 & 0.515 & $18.2(0.5)$ & $1.95(C$ & $30.2(2.4)$ & 74.5 & 1.1 & 3.7 & 3.1 & 8.3 \\
\hline 50 & 0.518 & $35.2(0.5)$ & $1.38(0.10)$ & $46.8(3.8)$ & 109.2 & 0.8 & 3.7 & 3.1 & 8.3 \\
\hline 51 & 0.532 & $52.8(0.5)$ & $1.01(0.08)$ & $50.0(4.0)$ & 92.8 & 0.7 & 3.6 & 3.1 & 8.2 \\
\hline 52 & 0.787 & $33.0(1.0)$ & $0.80(0.06)$ & $76.8(6.3)$ & 123.2 & 0.5 & 3.0 & 3.2 & 8.1 \\
\hline 53 & 0.781 & $33.7(0.5)$ & $1.19(0.09)$ & $57.1(4.7)$ & 118.4 & 1.1 & 3.1 & 3.5 & 8.3 \\
\hline \multicolumn{10}{|c|}{$T=(500 \pm 6) \mathrm{K}$} \\
\hline 54 & 0.425 & $35.0(1.0)$ & 0.8 & 58.9 & 130.0 & 0.6 & 3.1 & 3.2 & 8.2 \\
\hline \multicolumn{10}{|c|}{$T=(550 \pm 6) \mathrm{K}$} \\
\hline 55 & 0.201 & $33.7(0.5)$ & $1.25(0.10)$ & $30.2(2.5)$ & 113.3 & 1.6 & 3.5 & 3.5 & 8.5 \\
\hline 56 & 0.293 & $17.9(0.5)$ & $1.92(0$ & 23.1(1.9) & 73.5 & 1.1 & 3.5 & 3.1 & 8.2 \\
\hline 57 & 0.285 & $36.1(0.5)$ & $1.14(0.09)$ & $42.8(3.5)$ & 130.1 & 0.9 & 3.6 & 3.1 & 8.2 \\
\hline 58 & 0.287 & $52.0(0.5)$ & $1.07(0.08)$ & $43.3(3.5)$ & 135.6 & 0.9 & 3.5 & 3.1 & 8.2 \\
\hline 59 & 0.292 & $68.8(0.5)$ & $1.06(0.08)$ & $38.7(3.1)$ & 104.3 & 0.8 & 3.5 & 3.1 & 8.2 \\
\hline 60 & 0.407 & $15.4(0.5)$ & $0.93(0.07)$ & $37.4(3.0)$ & 66.1 & 0.9 & 3.4 & 3.1 & 8.2 \\
\hline 61 & 0.399 & $32.7(0.5)$ & $0.97(0.07)$ & $35.4(2.9)$ & 128.7 & 0.7 & 3.5 & 3.1 & 8.2 \\
\hline 62 & 0.383 & $47.7(0.8)$ & $0.87(0.07)$ & $56.3(4.6)$ & 133.1 & 0.7 & 3.6 & 3.1 & 8.2 \\
\hline 63 & 0.390 & $68.5(0.5)$ & $1.00(0 .($ & $45.8(3.7)$ & 103.5 & 0.9 & 3.4 & 3.1 & 8.2 \\
\hline 64 & 0.505 & $18.2(0.5)$ & $1.81(0.14)$ & $32.4(2.6)$ & 79.8 & 1.1 & 3.8 & 3.1 & 8.4 \\
\hline 65 & 0.490 & $35.2(0.5)$ & $1.25(0.09)$ & $50.3(4.1)$ & 138.0 & 0.7 & 3.9 & 3.1 & 8.4 \\
\hline 66 & 0.502 & $52.8(0.5)$ & $0.93(0.07)$ & $62.4(5.1)$ & 141.8 & 1.2 & 3.8 & 3.1 & 8.4 \\
\hline 67 & 0.604 & $51.5(0.5)$ & $0.93(0.07)$ & $68.0(5.5)$ & 142.1 & 1.1 & 3.8 & 3.1 & 8.4 \\
\hline \multicolumn{10}{|c|}{$T=(635 \pm 6) \mathrm{K}$} \\
\hline 68 & 0.597 & $51.5(0.5)$ & 0.9 & 5) & 155.5 & 0.5 & 4.0 & 3.1 & 8.4 \\
\hline \multicolumn{10}{|c|}{$T=(800 \pm 10) \mathrm{K}$} \\
\hline 69 & 0.191 & $33.7(0.5)$ & $1.28(0.10)$ & $36.2(3.0)$ & 134.9 & 1.8 & 3.9 & 3.5 & 8.7 \\
\hline 70 & 0.279 & $17.9(0.5)$ & $1.88(0.14)$ & $23.6(1.9)$ & 78.8 & 1.2 & 4.3 & 3.1 & 8.6 \\
\hline 71 & 0.275 & $36.1(0.5)$ & $1.13(0.09)$ & $40.2(3.3)$ & 150.1 & 0.9 & 4.4 & 3.1 & 8.6 \\
\hline 72 & 0.278 & $52.0(0.5)$ & $1.16(0.09)$ & $46.8(3.8)$ & 165.2 & 0.8 & 4.0 & 3.1 & 8.4 \\
\hline 73 & 0.278 & $68.8(0.5)$ & $1.24(0.09)$ & $39.7(3.2)$ & 139.9 & 0.9 & 4.0 & 3.1 & 8.4 \\
\hline 74 & 0.410 & $18.2(0.5)$ & $1.93(0.15)$ & $29.8(2.4)$ & 84.5 & 1.0 & 4.0 & 3.1 & 8.5 \\
\hline 75 & 0.400 & $35.0(0.5)$ & $0.92(0.07)$ & $60.2(4.9)$ & 152.0 & 0.6 & 4.0 & 3.2 & 8.5 \\
\hline 76 & 0.385 & $35.2(0.5)$ & $1.50(0.11)$ & $45.6(3.7)$ & 150.3 & 1.0 & 4.3 & 3.1 & 8.6 \\
\hline 77 & 0.405 & $51.5(0.5)$ & $1.23(0.09)$ & $51.9(4.2)$ & 164.8 & 0.6 & 4.0 & 3.1 & 8.4 \\
\hline
\end{tabular}


Continuation of Table 1

\begin{tabular}{|c|c|c|c|c|c|c|c|c|c|}
\hline \multirow[b]{2}{*}{ No. } & \multicolumn{2}{|c|}{ Conditions } & \multirow[b]{2}{*}{$\begin{array}{l}\omega, \\
\%\end{array}$} & \multirow[b]{2}{*}{$Y_{n}$} & \multirow[b]{2}{*}{$\begin{array}{c}\lambda_{c} \\
\mu \mathrm{s}^{-1}\end{array}$} & \multicolumn{4}{|c|}{ Error contributions (\%) } \\
\hline & $\begin{array}{c}\varphi, \\
\text { LHD }\end{array}$ & $\begin{array}{c}C_{t}, \\
\%\end{array}$ & & & & Stat. & $\Delta \varphi$ & $\Delta q$ & Total \\
\hline 78 & 0.375 & $68.5(0.5)$ & $1.25(0.09)$ & $47.0(3.8)$ & 145.0 & 0.8 & 3.9 & 3.1 & 8.4 \\
\hline 79 & 0.484 & $18.2(0.5)$ & $1.84(0.14)$ & $32.7(2.6)$ & 84.2 & 0.9 & 4.3 & 3.1 & 8.6 \\
\hline 80 & 0.484 & $35.2(0.5)$ & $1.29(0.10)$ & $50.2(4.1)$ & 155.3 & 0.7 & 4.3 & 3.1 & 8.6 \\
\hline 81 & 0.491 & $51.5(0.5)$ & $1.14(0.09)$ & $59.4(4.8)$ & 173.0 & 0.6 & 4.3 & 3.1 & 8.6 \\
\hline
\end{tabular}

3.1. Temperature and Pressure Control. Temperature of liquid $D / T$ was determined by measuring the $D / T$ vapor pressure with the tensometric gauges having the accuracy $0.5 \%$. So, the $D / T$ temperature was determined with an accuracy $0.1 \mathrm{~K}$. Temperature of gaseous $D / T$ was measured by special thermocouples. During the experimental runs a small temperature gradient existed in the $D / T$ mixture, which was taken into account in temperature and its error determination. An accuracy of temperature knowing was $3-10 \mathrm{~K}$ in the $40-800 \mathrm{~K}$ range. Pressure was measured with the use of strain pressure gauges having the calibration error $3 \%$.

3.2. $D / T$ Mixture Density. Nuclear density of liquid $D / T$ was determined using the cryogenic data on deuterium and tritium [51] taking into account the mixture content. Errors in density were of $2 \%$.

Nuclear density of gaseous $D / T$ was determined by two ways. The first one was the use of the deuterium (tritium) equation of state [52] where the gas temperature and pressure known from measurements. Some corrections for the presence of a buffer volume having room temperature was made. The other way was the density determination via the quantity of gas in the target of known volume. Both methods gave identical results within accuracy of $4 \%$. The final error of gas density was up to $3-4 \%$.

In addition, we have another way to check the mixture density. If the muon beam intensity is stable, the number of muon stops in the mixture per time unit is proportional to the mixture density. In several cases we made some corrections (about few percent) to the mixture density based on this method.

3.3. Measurements of Isotope and Molecular Gas Composition. The chromatographical method [53] was used to control the isotope and molecular composition of the mixtures. In addition, to obtain the $D / T$ and $T_{2}$ content the ionization chamber was used. Measurements were made before filling the target and after evacuation of the mixture from it.

The chromatographical analysis showed the molecular compositions very closely to the equilibrium ones

$$
C_{D D}: C_{D T}: C_{T T}=C_{d}^{2}: 2 C_{d} C_{t}: C_{t}^{2}, \quad C_{t}+C_{d}=1
$$


for each gaseous mixture exposed to a muon beam. However, for the liquid mixtures the molecular content can differ from the equilibrium due to the dynamic effects in evaporation of multi-component liquid, investigated by us under the conditions of our target in [54]. The deviation from the equilibrium state becomes noticeable for the high tritium concentration $C_{t}>50 \%$. Appropriate corrections to the molecular and isotope concentrations of the liquid mixture were made in [55]. Note that quantity of protium in $D / T$ mixtures did not exceed $1 \%$.

3.4. $D / T$ Mixture Purity and ${ }^{3}$ He Accumulation. As follows from Eq. (10), the expression for the cycling rate does not depend on the muon loss including as a part the effect of impurities with $Z>1$. Contrary to this, the muon losses depend on the cycling rate $\left(\lambda_{d t \mu}\right.$ and $\left.\lambda_{d t}\right)$. The larger the cycling rate the closer the muon losses are to their natural limit equal to the probability $\omega_{s}$.

As is seen from Eq. (11), the muon transfer to the possible impurities influences on the value of $\omega$. That is why one should provide the impurity level as small as possible. Actually, one should provide the condition

$$
\lambda_{Z} \cdot C_{Z}<<\omega_{s} \cdot \lambda_{c} .
$$

It is necessary to distinguish two sorts of impurities: impurities with $Z>2$ and He admixtures.

3.4.1. Impurities with $Z>2$ and ${ }^{4} \mathrm{He}$. They are predominately helium-4, carbon, oxygen and nitrogen originating from the imperfect purification of the mixture before filling the target and removing the residual gaseous elements from the target walls during an exposure.

The special preparation system based on palladium filters [46] provides filling of a target with gas purified of impurities at the level $C_{Z}<10^{-7}$ of volume parts. As the outgassing effect increased with temperature, the mixture purity varied from $C_{Z}<10^{-7}$ for $T=20 \mathrm{~K}$ to $C_{Z}=10^{-5}-10^{-6}$ for $T=800 \mathrm{~K}$. The rate of the muon transfer from the $t \mu$ atom to the pointed admixtures is $\lambda_{Z} \sim 10^{11} \mathrm{~s}^{-1}$ for nuclei with $Z>2$ [56] and $\lambda_{4} \mathrm{He} \sim(1-5) \cdot 10^{9} \mathrm{~s}^{-1}$ for ${ }^{4} \mathrm{He}[57,58]$. Therefore condition (12) is satisfied only for a liquid $D / T$ mixture where the cycling rate is rather high $\left(\lambda_{c}=50-120 \mu \mathrm{s}^{-1}\right.$ depending on the tritium concentration) and most impurities (excluding helium) are solid and freezed out on the target walls.

3.4.2. ${ }^{3} \mathrm{He}$ admixture. The tritium handling system provides the initial concentration $C_{\mathrm{He}}$ of ${ }^{3} \mathrm{He}$ in the mixture before pouring into a target at the level $10^{-7}$. However, due to the tritium $\beta$-decay, ${ }^{3} \mathrm{He}$ is accumulated in a target according to relation

$$
C_{\mathrm{He}}(\tau)=C_{t} \cdot\left[1-\exp \left(-\lambda_{\text {trit }} \cdot \tau\right)\right]
$$

where $\lambda_{\text {trit }}=6.4 \cdot 10^{-6} \mathrm{~h}^{-1}$ is the tritium decay rate. Hence, the process of the muon transfer from the $t \mu$ atom to ${ }^{3} \mathrm{He}$ (with the rate $\lambda^{3} \mathrm{He} \sim 2 \cdot 10^{8} \mathrm{~s}^{-1}$ [57]) can essentially influence the muon losses. 
${ }^{3} \mathrm{He}$ accumulation effect is quite different for liquid and gaseous $D / T$. It was shown in experiment [58] that ${ }^{3} \mathrm{He}$ in liquid $D / T$ diffuses and goes out to the vapor gas. Our cooling system of the LTT [43] provided the passage of all the $D / T$ mixture through the vapor phase during $\sim 1 \mathrm{~h}$, which led to the ${ }^{3} \mathrm{He}$ escape out of liquid $D / T$. So in experiments with liquid $D / T$ we had no problem with $D / T$ purity.

In experiments with gaseous $D / T$ we were forced to refill the target every $10-40 \mathrm{~h}$ (depending on the tritium content in $D / T$ ) to avoid accumulation of ${ }^{3} \mathrm{He}$ larger than the «critical value» $C_{3} \mathrm{He}=10^{-5}$.

\section{DATA TREATMENT}

The data processing included the following stages.

1. Selection of events. By an event is meant occurrence of the processes caused by a single muon beginning with the muon stop in the target and ending with the muon decay. An example of the event as seen by the detectors is presented in Fig. 7. The most important criteria for the event to be accepted was presence of a reliable signal for the $\mu$-decay electron.

2. Creation of the charge and time spectra for neutrons from the $d+t$ reaction and electrons from the $\mu$ decay.

3. Fit of these spectra to determine the «effective» MCF parameters $\lambda_{c}, \omega$ and $Y_{n}$.

4. Analysis of these parameters as functions of the tritium concentration to obtain the $d t \mu$ formation rates $\lambda_{d t \mu-d}, \lambda_{d t \mu-t}$ and the muon-to-helium sticking probability $\omega_{s}$.

4.1. Analysis Methods. The most popular and practically the only method used by most groups involved in the study of the MCF $d+t$ process is the so-called standard method where the yield and time distribution of all detected neutrons from reactions (5), (6) are registered and analyzed. This distribution has the well-known one-exponent form (8). The number of $\mu$-decay electrons $N_{e}$ is used for normalization

$$
N_{n} / N_{e}=\epsilon_{n} \Lambda_{c} /\left[\lambda_{0}+\omega \Lambda_{c}\right] .
$$

The slope of exponent (8) $\lambda_{n}$ and the normalized neutron yield $Y_{n}$ are the measured parameters. The values of $\Lambda_{c}, \omega$ and $Y_{n}$ are extracted from (8), (9) and (13):

$$
\epsilon_{n} \varphi \lambda_{c}=\frac{N_{n}}{N_{e}} \cdot \lambda_{n}, \quad \frac{\omega}{\epsilon_{n}}=\frac{\lambda_{n}-\lambda_{0}}{\epsilon_{n} \varphi \lambda_{c}}, \quad \epsilon_{n} Y_{n}=\frac{N_{n}}{N_{e}} .
$$

In the Dubna experiments we also used standard method. To obtain spectrum (8) we created the time distribution of the neutron detector charge $Q(t)$. For this we summed the amplitude spectra for each neutron detectors ND1 and ND2. Then the 
spectrum $Q(t)$ was transformed to the time distribution of the number of events $N_{n}(t)=Q(t) / \bar{q}$ using the unit charge $\bar{q}$ [59]. The latter was measured under special conditions providing a low neutron multiplicity, where each charge pulse corresponded to one neutron. Charge distributions obtained in such exposures were compared with the calculated ones to obtain the experimental value of $\epsilon_{n}$ as a function of the threshold.

The number of electrons $N_{e}$ was obtained from the analysis of the electron time spectra $N_{e}(t)$ using the distribution $B_{\text {empty }}(t)$ measured with an empty target:

$$
N_{e}^{\text {total }}(t)=k \cdot B_{\text {empty }}(t)+A_{e} \cdot \exp \left(-\lambda_{e} t\right)+F,
$$

where $\lambda_{e}$ is the muon disappearance rate, $F$ is the accidental background. In this fit $k, A_{e}, \lambda_{e}$ and $F$ are parameters. The observed muon disappearance rates $\lambda_{e}$ are close to the muon decay rate $\lambda_{0}=0.455 \mu \mathrm{s}^{-1}$ and depend on the mixture purity. In exposures with liquid $D / T$, where the purity is maximum, $\lambda_{e}$ is obtained equal to $\lambda_{0}$ within $1 \%$.

A typical example of the fitted time distributions of decay electrons and fusion neutrons for the $D / T$ filled target is shown in Fig. 8. The dashed line corresponds to the electrons from decays of muons stopped in the target walls (empty target).
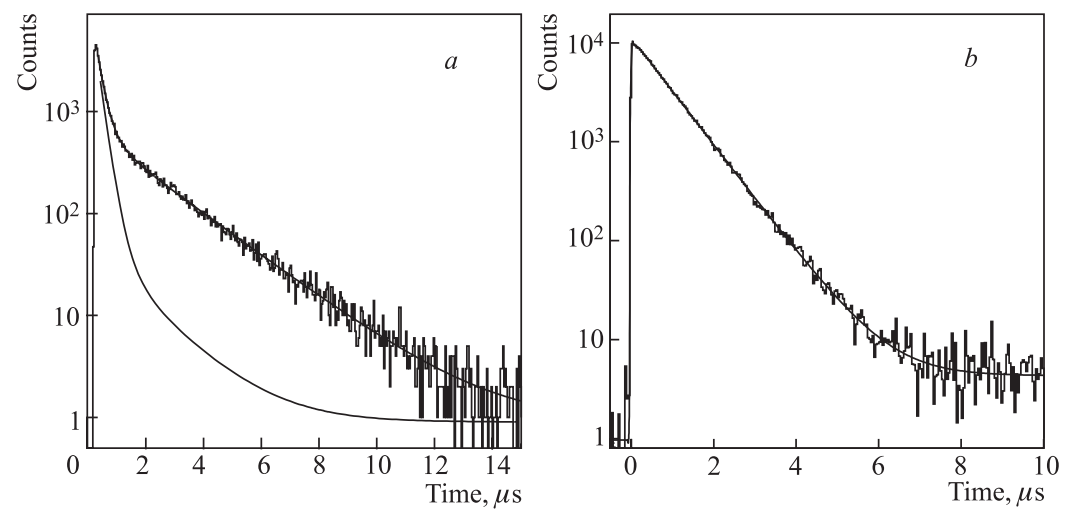

Fig. 8. Example of electron $(a)$ and neutron $(b)$ time distributions. Solid lines are the optimum fits with expressions (15) (a) and (8) (b), the dashed line is the electrons from empty target

The principal disadvantage of the standard method is that the main MCF parameters - cycling rate and effective muon losses - are not obtained directly, only their product is measured directly. In our measurements we employed two novel independent methods proposed and developed in Dubna [60, 61]. These analysis methods make it possible to directly measure the values of $\lambda_{c}$ and $\omega$. 
A proposal of [60] was to measure the distribution $N_{n e}(t)$ which was a function of the interval $t=t_{e}-t_{n}$ between the last detected neutron of the series and the $\mu$-decay electron. This distribution has the form of a sum of two exponents with significantly different slopes [60,61].

$$
d N_{n e} / d t=\left(\lambda_{0} / \lambda_{n}\right) \cdot\left[\omega \cdot \Lambda_{c} \cdot \exp \left(-\lambda_{0} \cdot t\right)+\epsilon_{n} \cdot \Lambda_{c} \cdot(1-\omega) \cdot \exp \left(-\left(\lambda_{0}+\lambda_{n}\right) \cdot t\right)\right]
$$

where $\lambda_{n}$ is expressed as

$$
\lambda_{n}=\left(\epsilon_{n}+\omega-\epsilon_{n} \cdot \omega\right) \cdot \Lambda_{c}
$$

The first («slow») exponent corresponds to the events with muon sticking and the second («fast») one to the events without sticking. The cycling rate is determined from the fast component slope, and the muon loss is obtained from the ratio between the amplitudes of the slow and fast exponents: $A_{s} / A_{f}=\omega / \epsilon_{n}(1-\omega)$. The examples of such distributions obtained in a liquid $D / T$ mixture are presented in Fig. 9. As is seen from the figures, the events with and without sticking are clearly separated. Different slopes of the fast components of the spectra reflects the different values of the cycling rate realized for the tritium concentrations $C_{t}=35.2 \%$ and $C_{t}=85.5 \%$. The advantage of the method is that charge calibration is not necessary in this case.
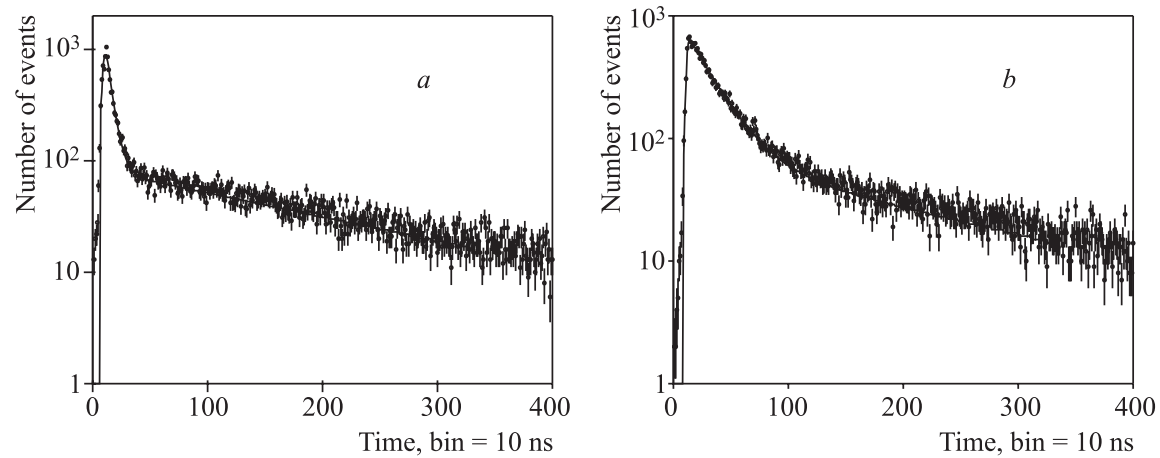

Fig. 9. Electron - last neutron timing spectra measured with a liquid $D / T$ mixture. $a$ - spectrum corresponds to the exposure with $C_{t}=35.2 \%$ and $b-$ selected for $C_{t}=85.5 \%$. Lines are the fits with expressions (16), (17) and the optimum parameters $\epsilon_{n} \Lambda_{c}$ and $\omega / \epsilon_{n}$

Another idea [61] was to measure the neutron multiplicity (number of detected neutrons, $k$, per muon) distribution in some definite interval $T$. If one selects the events for which the muon does not decay in this interval, then this distribution would be a sum of two terms. One of them, the Gaussian (Poisson) with the mean 
$m=\epsilon_{n} \Lambda_{c} \cdot T$, corresponds to the events without sticking, and the other, depending on $\omega$ and falling with $k$, is the distribution of events with muon sticking.

The rigorous expression for the multiplicity distribution was obtained in [62]. It has the form

$$
f(k)=\frac{\left[\epsilon_{n}(1-\omega)\right]^{k}}{\left(\epsilon_{n}+\omega-\epsilon_{n} \omega\right)^{k}} \cdot P(k)+\frac{\left[\epsilon_{n}(1-\omega)\right]^{k-1} \omega}{\left(\epsilon_{n}+\omega-\epsilon_{n} \omega\right)^{k}} \cdot F(k),
$$

where $P(k)$ is the Poisson distribution with the mean $m=\lambda_{n} T$

$$
P(m)=\frac{\left(\lambda_{n} T\right)^{k}}{k !} \cdot \mathrm{e}^{-\lambda_{n} T}, \quad F(k)=1-\mathrm{e}^{-\lambda_{n} T} \sum_{i=0}^{k-1} \frac{\left(\lambda_{n} T\right)^{i}}{i !},
$$

and $\lambda_{n}$ is given by formula (17).

Formula (18) corresponds to the «event mode» where the number of detected neutrons was considered. Really the distributions of the neutron detector charge are measured in experiments and were divided by the unit charge to obtain a multiplicity distribution. The real response function of the detector results in diffusion of the measured spectra as compared with the ones obtained in the «event mode». It turned out that in good approximation (with accuracy 2-3\% in cycling rate) the real distribution might be obtained as convolution of formula (18) with the Gaussian function. The Gaussian width is varied to obtain the best agreement between the experiment and calculations.

We can also select only non-sticking events. For this, one should exclude the requirement of the electron signal in the trigger and select only those neutron series whose duration was larger than the chosen interval $T$. Plotting and analyzing the multiplicity distribution of such events we can directly obtain the cycling rate. The advantage of this method is that we do not need the $\mu$-decay electron existence fact. Of course, only $\lambda_{c}$ can be determined in this case since events without muon sticking to helium are accepted. The examples of fitted multiplicity distributions are presented in Fig. 10.

The comparison of all methods used by us in the analysis is given in the Table 2.

Table 2: Comparison of different methods used in the MCF study

\begin{tabular}{c|c|c|c}
\hline Method & $\begin{array}{c}\lambda_{c}, \omega \\
\text { determination }\end{array}$ & $\begin{array}{c}\text { Charge } \\
\text { calibration }\end{array}$ & $\begin{array}{c}\text { Electron } \\
\text { detection }\end{array}$ \\
\hline Standard & Indirect & Necessary & Necessary \\
\hline$t_{e}-t_{n}$ & Direct & Not necessary & Necessary \\
\hline Multiplicity & Direct & Necessary & Not necessary \\
\hline
\end{tabular}

The statistical power is practically the same for all methods. Indeed, in the standard method the main factor for the statistical accuracy is the limited number 

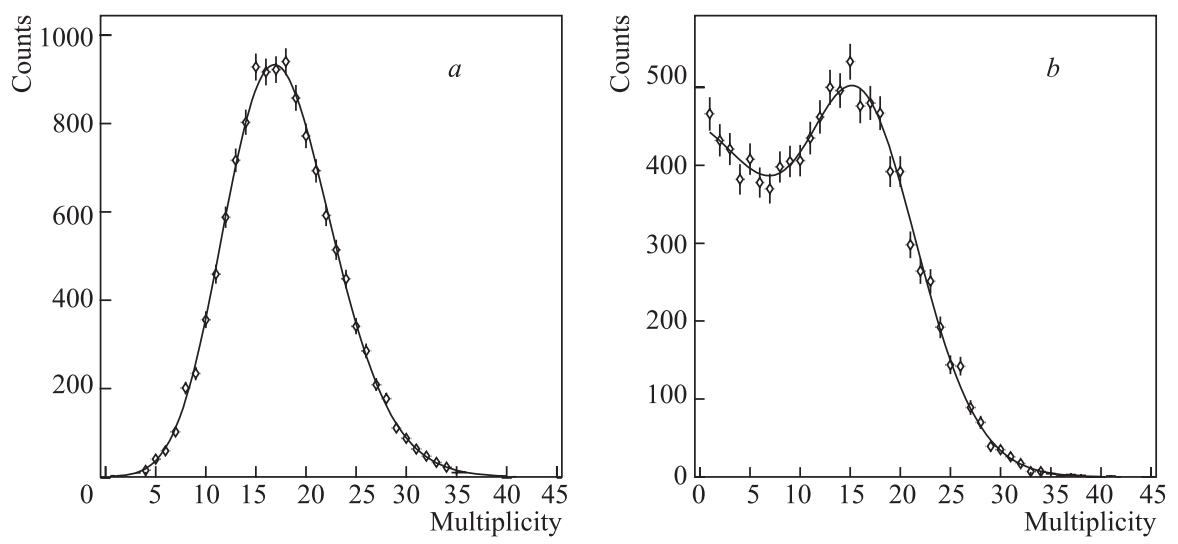

Fig. 10. Multiplicity distributions in time interval $T=1 \mu \mathrm{s} . \quad a-$ distribution without sticking events, $b$ - with sticking events. Curves are the optimum fits

of electrons, the number of neutrons is much higher under real experimental conditions. In two other methods the full statistics is the number of the first or last neutrons which are also approximately equal to the electron number.

In our investigations we use all three methods mentioned. It allows us to realize the reliable data analysis with minimum systematic uncertainties. Of course, the full analysis is rather complicated and includes many tests with different selection rules for events to be accepted.

4.2. Electron Identification. A serious problem in the MCF data analysis is how to distinguish the real electron from the false one. Under the conditions where one muon can cause up to 100 reactions (5) it is possible to detect a neutron by the electron detector and accept it as an electron. Contrary to the measurements of other groups, we detect electrons with a proportional wire counter, having a very low sensitivity to neutrons. However, even in this case the fraction of the false electrons caused by the neutron counts was noticeable.

Only the last (in time) electron signal is accepted as real. It will be enough to exclude the false electrons if the electron detection efficiency would be $\epsilon_{e}=$ $100 \%$. However, for different reasons (see [63]) this efficiency is not equal to unity. Thus the situation can occur where the real electron is not detected and the false one is interpreted as real. The presence of false electrons results in distortion of $\lambda_{c}, \omega$ and $Y_{n}$. The cycling rate determined according to formula (14) is distorted due to the error in $N_{n}, N_{e}$, and $\lambda_{n}$ extracted from expressions (8), (15) feels the error in $\lambda_{c}$. On the other hand, the confusion of the real and false electrons leads to distortion in the relation between «stick» series (interrupted due to the muon sticking) and «unstick» series (ending with $\mu$ decay). The latter are accepted more effectively. Thus the results for the muon losses are also distorted. Finally, the distortion of the slope of the electron time distribution does not make 
it possible to correct the estimate of the $D / T$ mixture purity, and thus to check the parameters of the purification system.

Fortunately, the cycling rate determined from the peak position in the multiplicity spectrum is free of false electrons. It is a very important circumstance allowing reliable data on the cycling rate serving as a source of the «elementary» process parameters such as the $d t \mu$-molecule formation rate. Of course, it is very desirable to obtain a correct value for $\lambda_{c}$ by different independent methods. Moreover, getting correct data on the muon losses is an independent important task.

4.2.1. Selection by the energy loss in neutron detector. The effective way to reject false electrons was elaborated and used in our work [23]. For this we required the following when selecting events.

1. Electron signals from the PC and ND1 or ND2 should coincide.

2. Energy which the electron releases in the neutron detector should be more than the maximum possible energy released by a $14 \mathrm{MeV}$ neutron in this detector. This allows reliable discrimination of false electrons. The use of these selection criteria allowed us to obtain the data on $\lambda_{c}$ and $\omega$ coinciding for all three analysis methods within $5 \%$ [23].

The disadvantage of this selection is a decrease in the statistics because an essential part of the useful events are rejected. This decrease becomes much more sensitive in experiments with a high-pressure gaseous target having rather thick walls, for which the «output» electron energy spectra are noticeably distorted and the transparency of the target walls for electrons is noticeably smaller than for the liquid target. So for a gaseous target reliable neutron-electron separation was connected with larger statistics losses than that for a liquid target.

4.2.2. Selection by the time position of the electron signal relative to neutron series. Not to lose statistics we developed a new method [63] for the false electron discrimination which is most effective for the large neutron detection efficiency realized in our experiments. Now we put the criterion «electron inside neutron series». For this we consider the neutron detector charge $Q$ (sum of amplitudes) on some time interval $(\Delta T)$ close to the electron signal and delayed relative to it by $\Delta t$. The events were accepted under the condition that the charge $Q$ is smaller than the threshold: $Q<Q_{\mathrm{th}}$. Our considerations [63] shows that the proper values are $\Delta t=60 \mathrm{~ns}$ and $\Delta T=500 \mathrm{~ns}$. The largest values of $Q_{\mathrm{th}}$ correspond to events without selection for the false electron. In this case the distortion in the electron yield and time spectrum (15) is the largest. The opposite case (low $Q_{\mathrm{th}}$ ) corresponds to the smallest distortions for electrons and to the minimum value of the electron time slope $\lambda_{e}$ which practically coincides with the one determined using selection by the electron energy in neutron detector.

Opposite situation occurs for the slope of the neutron time distribution (8). In the case where the real electron is not detected, the false one is accepted as electron. It means that the long neutron series are predominately detected 
because appearance of a false electron is most probable right in those series. Indeed, our considerations [63] show that the minimum $Q_{\text {th }}$ (maximum false electron rejection) leads to the maximum slope $\lambda_{n}$. Again, the «correct» value of $\lambda_{n}$ is in agreement with the one obtained with selection by electron energy in the neutron detector.

The main MCF parameters obtained under two different selection options coincide within an accuracy $3-4 \%$. The reliability of the data is confirmed by the fact that the value of the cycling rate determined by the standard method is identical to the one yielded by the multiplicity method where it does not depend on the selection criteria. The method considered gives the statistics 4-5 times larger than in the case with energy discrimination (Sec. 4.2.1). It indicates that we could find the way described in detail in [63] for correctly obtaining the MCF parameters without essential loss in statistics.

4.3. Neutron Detection Efficiency. All methods discussed above give the values of $\lambda_{c} \cdot \epsilon_{n}$ and $\omega / \epsilon_{n}$. To obtain the MCF parameters $\lambda_{c}$ and $\omega$ one should know the neutron detection efficiency $\epsilon_{n}$.

It is not a trivial task to determine $\epsilon_{n}$ for organic scintillation counters because it is influenced by factors like the geometry of the surrounding material, generation of light by various reaction products and because many energy-dependent crosssections are involved. Because of lack of neutron calibration sources with wellknown intensity and sufficiently large energy, the efficiency had to be calculated. The Monte Carlo technique was used.

Calculations of $\epsilon_{n}$ for neutrons detected by the ND in the Dubna experiments are described in [64]. The CERN package GEANT was used in [64] for the simulation calculations. Because it lacks the appropriate low and fast neutron interaction cross-sections, GEANT was linked with the MICAP package. MICAP uses experimental neutron cross-sections from the ENDF/B-VI data base from $20 \mathrm{MeV}$ down to thermal energies $\left(10^{-5} \mathrm{eV}\right)$. This includes partial cross-sections, angular distributions, energy distributions of reaction products and deexcitation photons. The preprocessed ENDF/B-VI data represent the experimental data within $2 \%$.

After the calculation of the energy deposited inside the scintillator, the electronic output signal was obtained by first converting the energy into scintillation light considering the particle type, and then converting the total light output into an electric signal by applying the detector response function [49]. This function takes into account such factors as non-uniform light collection depending on the position of light generation inside the scintillator and photon statistics. The results of calculations [64] for $14 \mathrm{MeV}$ neutrons from reactions (5), (6) are presented in Fig. 11 together with the measured spectra.

One neutron detected in a scintillator may generate a response from one detector or, due to scattering or to generated gamma rays, from both detectors. This leads to a single and coincident rate. The corresponding spectra are shown 


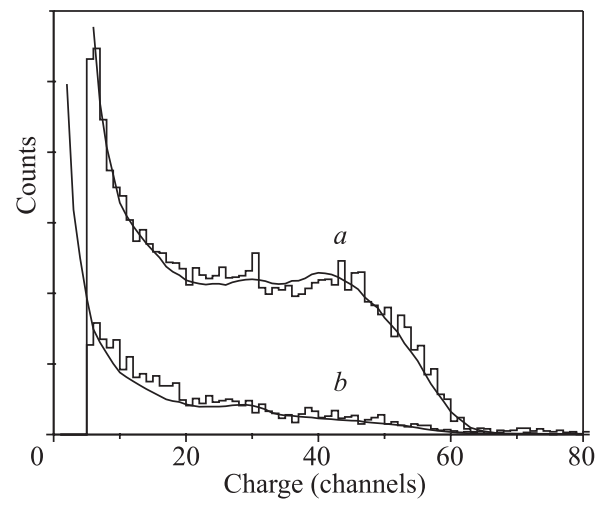

Fig. 11. Charge distribution for $14-\mathrm{MeV}$ neutrons measured in [23] with the ND (histograms). Distributions are plotted for single $(a)$ and coincident events $(b)$. Lines are the Monte Carlo calculations [64]

in Fig. 11 together with the measured ones. As seen, there is good agreement between the measurements and the calculations for both cases (single and coincident). The intensity and amplitude calibration of the calculated single spectrum was normalized to single data. The normalization thus obtained is then applied to the calculated coincident spectrum, which then neatly coincides with the corresponding data. This means that the single to coincident ratio is well predicted, which is considered to be a sensitive validation check for the calculations. The estimated relative uncertainty in $\epsilon_{n}$ is not worse than 5-7\%.

The problem is how the neutron detection efficiency depends on the neutron multiplicity (cycling rate). The main idea of using the flash ADC is that the total charge per number of neutrons is conserved even when the ND signals mostly overlap. However, it is true only for the zero charge threshold. Really, the cluster charge should be limited to reduce the low-energy background. At a high neutron multiplicity clusters of small charge can overlap with one or more others and hence can be accepted (non-effective threshold). Obviously, this results in an increase of the detection efficiency as compared with the low neutron multiplicity. The real increase depends on several factors, such as the shape of the ND signal, the form of the response function, the magnitude of the threshold and the measured cycling rate. Since one would expect an essential correction to the value of $\epsilon_{n}$, the problem required special consideration.

This was made in [65] where the fusion neutron registration was Monte Carlo simulated for a wide cycling rate. All three analysis methods were considered. It turned out that in the standard and multiplicity methods the corresponding corrections to the efficiency were not so large. So, even for the maximum possible measured cycling rate $\epsilon_{n} \Lambda_{c}=40 \mu \mathrm{s}^{-1}$ they are only $12 \%$. 


\section{RESULTS}

5.1. The Effective MCF Parameters. The effective MCF parameters $\lambda_{c}, \omega$ and $Y_{n}$ were obtained from the fit of the distributions considered in the «standard», «multiplicity» and «t $t_{e}-t_{n}$ » analysis methods. In spite of the fact that the first two methods are more reliable, the results obtained by three different methods were in agreement with an accuracy 3-4\%. They are presented in Table 1 and in Fig. 12.
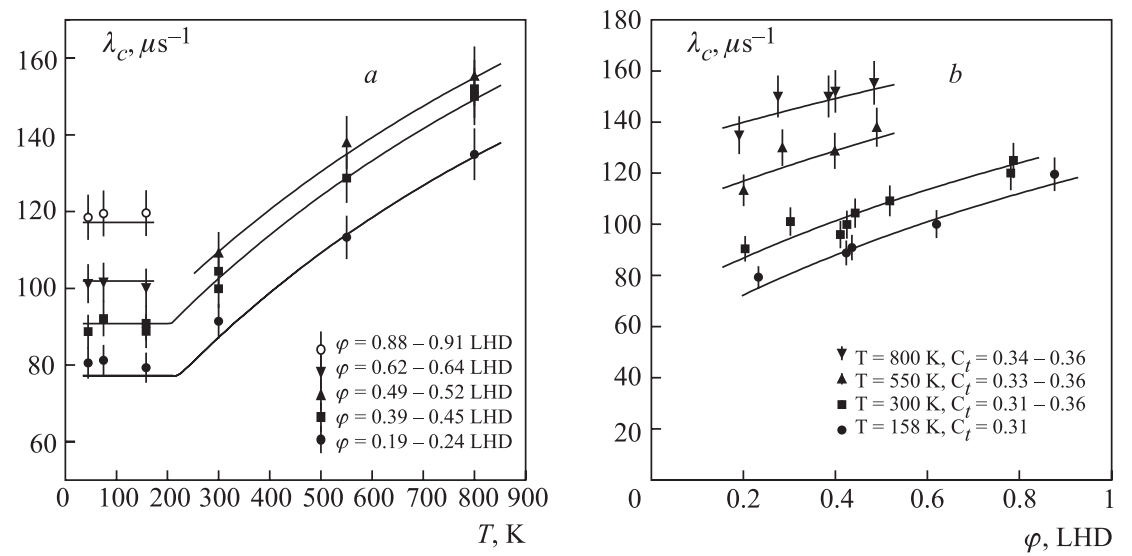

Fig. 12. $a-$ normalized cycling rates as a function of temperature for gaseous $D / T$ mixture at $C_{t} \simeq 33 \%$ and different densities. $b$ - normalized cycling rates as a function of density for gaseous $D / T$ mixture at $C_{t} \simeq 33 \%$ and different temperatures. The curves are obtained with optimum parameters

The statistical uncertainty in the results is determined by the number of events and the fit accuracy. In both main methods («standard» and «multiplicity») the statistics provides this error not higher than $2 \%$. Note that in the multiplicity method we do not need the muon number normalization.

The following factors contribute to the systematic error:

- Uncertainty in the neutron detection efficiency makes the maximum contribution to the systematic error. It was estimated from the calculation of $\epsilon_{n}$ and an accuracy in the energy threshold determination and is of $6 \%$ in total.

- Uncertainty of the charge calibration procedure gives an error smaller than $3 \%$.

- Uncertainty of the gas and liquid density (for normalized cycling rate) is about $3-4 \%$ and $2 \%$ respectively.

- Uncertainty of the time zero position (only for the standard method) gives a systematic error smaller than $0.5 \%$.

- Uncertainty due to the correct selection of muon decay electrons (see Sec. 4.2, only for the standard method) $2 \%$. 
- Uncertainty caused by possible instability of detectors and electronics does not exceed $2 \%$.

Then, the total uncertainty in the $\omega, Y_{n}$ and absolute values of $\lambda_{c}$ did not exceed $9 \%$. Obviously the relative dependencies of the cycling rate on temperature and density are known with a better accuracy (4.5-5.5\%).

5.2. The $d t \mu$-Molecule Formation Rate and Muon Sticking Probability.

The usual way to determine the «physical» values $\lambda_{d t \mu}$ and $\omega_{s}$ is an analysis of the «effective» parameters $\lambda_{c}$ and $\omega$ using expressions (10), (11), representing their dependence on tritium concentration and density. First of all, for this purpose it is necessary to express $q_{1 S}$ as a function of $C_{t}$ and $\varphi$. Taking into account general expression (1) and the theoretical predictions $[32,34,66]$ as well as experimental results $[17,35]$, the parametrization of $q_{1 S}$ was chosen in the form

$$
q_{1 S}\left(C_{t}, \varphi\right)=\frac{1}{1+(b+c \cdot \varphi) \cdot C_{t}} .
$$

5.2.1. Fit of the liquid $D / T$ data. Muon sticking probability $\omega_{s}$. As we noted the most expedient condition for $\omega_{s}$ measurement is a liquid $D / T$ mixture where $\lambda_{c}$ is high and the admixture content is negligible. Our first data for liquid $D / T$ mixture were given in [23]. In the present paper we corrected the values of the molecular concentrations and, besides, performed the common fit of $\lambda_{c}$ and $\omega$ in liquid $D / T$ using formulae (10), (11). As for all liquid points the density values were very close to each other, the parametrization for $q_{1 S}$ was chosen as

$$
q_{1 S}\left(C_{t}\right)=\left(1+a \cdot C_{t}\right)^{-1},
$$

where $a \equiv b+c \cdot \varphi$ from (19). We perform a set of fits varying different parameters according to the known theoretical and experimental data for $\lambda_{1-0}$ [68, 69], $\lambda_{t t \mu}[70,71,72]$ and $\lambda_{d t \mu-t}[17,18,70,14]$. Such variation does not lead to the significant change in the results. The systematic error $\Delta \epsilon_{n}$ (the same for all liquid data) was excluded from the data errors in this fits. In Table 3 the values used for the MCF cycle parameters and the results of one of the fits are shown.

The fit results are shown in Fig. 13 and in Table 3 . The main results for the liquid ( $T \simeq 22 \mathrm{~K}, \varphi \simeq 1.22 \mathrm{LHD}) D / T$ mixture are

$$
\begin{gathered}
\lambda_{d t \mu-d}=\left(685 \pm 35^{\text {stat }} \pm 41^{\text {syst }}\right) \mu \mathrm{s}^{-1}, \\
\lambda_{d t \mu-t}=\left(18 \pm 6^{\text {stat }} \pm 11^{\text {syst }}\right) \mu \mathrm{s}^{-1}, \\
\omega_{s}=\left(0.573 \pm 0.021^{\text {stat }} \pm 0.032^{\text {syst }}\right) \% .
\end{gathered}
$$

Our value of $\lambda_{d t \mu-d}$ (21) is essentially higher than the PSI group data [14] but in agreement with the LAMPF results [17] (see Fig. 15). The value $\lambda_{d t \mu-t}$ 
(22) is in satisfactory agreement with those obtained in [17, 18] $\lambda_{d t \mu-t}=20 \mu \mathrm{s}^{-1}$ and [70] $\lambda_{d t \mu-t}=11_{-11}^{+6} \mu \mathrm{s}^{-1}$. Unexpected high rate $\lambda_{d t \mu-t}=160 \mu \mathrm{s}^{-1}$ was obtained by the RIKEN group [16, 73]. Fixing this value we do not achieve any satisfactory agreement of fit to our data, so we conclude that this value is uprated by about a factor of 5 .

Table 3: Results of one from the set of common fits of the data for liquid $D / T$. Fixed parameters are given with references

\begin{tabular}{ll|ll}
\hline \multicolumn{2}{c|}{ Parameter } & Value & \\
\hline $\mathrm{a}$ & & $2.9 \pm 0.4$ & free \\
$\lambda_{Z} C_{Z}$ & $\mu \mathrm{s}^{-1}$ & $0.08 \pm 0.03$ & free \\
$\lambda_{d t}$ & $\mu \mathrm{s}^{-1}$ & 280 & {$[18,21,35,36]$} \\
$\lambda_{1-0}$ & $\mu \mathrm{s}^{-1}$ & 1200 & {$[68,69]$} \\
$\lambda_{d d \mu}^{3 / 2}$ & $\mu \mathrm{s}^{-1}$ & 3.5 & {$[11]$} \\
$\lambda_{3 / 2-1 / 2}$ & $\mu \mathrm{s}^{-1}$ & 36 & {$[11]$} \\
$\lambda_{f}^{t t}$ & $\mu \mathrm{s}^{-1}$ & 14 & {$[72]$} \\
$\omega_{d d}$ & $\%$ & 0.13 & {$[11]$} \\
$r$ & & 0.51 & {$[11]$} \\
$\lambda_{t t \mu} \omega_{t t}$ & $\mu \mathrm{s}^{-1}$ & $0.28 \pm 0.15$ & free \\
$\lambda_{d t \mu-d}$ & $\mu \mathrm{s}^{-1}$ & $650 \pm 40$ & free \\
$\lambda_{d t \mu-t}$ & $\mu \mathrm{s}^{-1}$ & $21 \pm 8$ & free \\
$\omega_{s}$ & $\%$ & $0.574 \pm 0.022$ & free \\
\hline
\end{tabular}
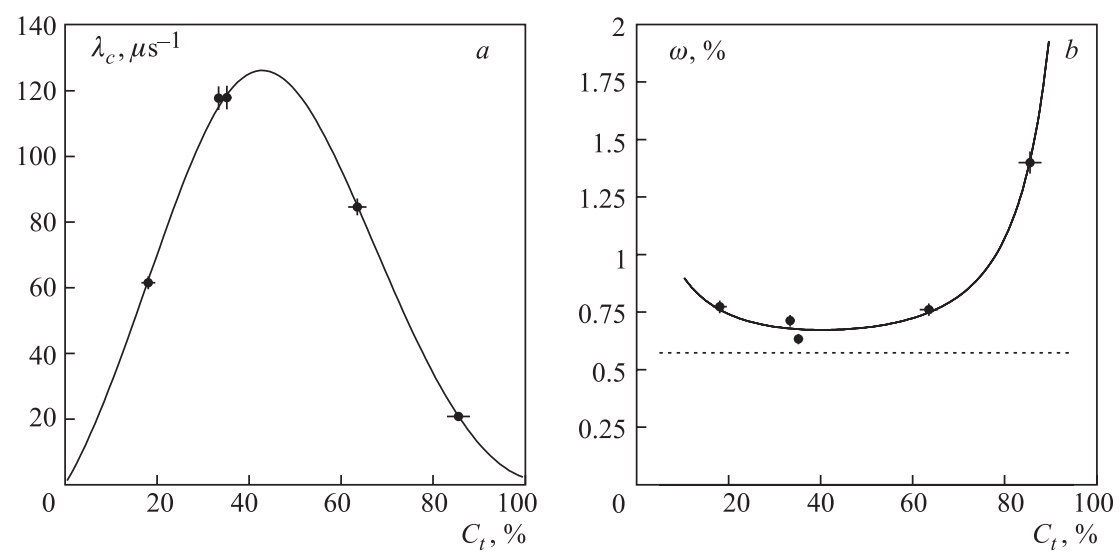

Fig. 13. Normalized cycling rates $(a)$ and muon loss probability $(b)$ as a function of the tritium concentration for the liquid $D / T$ mixture $(T=22 \mathrm{~K}, \varphi \simeq 1.22 \mathrm{LHD}$ ). Solid lines are optimum fits. The dashed line is value (23) 
The probability $\omega_{s}$ of effective muon-to-helium sticking in $d t$ fusion is one of the most important MCF characteristics, because it limits the number of fusions per muon. In theory the $\omega_{s}$ is considered as the product $\omega_{s}=\omega_{s}^{0} \cdot(1-R)$, where $\omega_{s}^{0}$ is the «initial» sticking probability directly after fusion and $R$ is the probability of muon-from-helium stripping during the $\mathrm{He} \mu$ thermalization stage. $R$ is density dependent and so for $\omega_{s}$ theory predicts the slow, close to linear, decrease with density. Comparison of different theoretical and experimental results on the $\omega_{s}$ is presented in Table 4.

Table 4: Comparison of the results on the $\omega_{s}$ obtained in different experimental and theoretical investigations

\begin{tabular}{l|l|l}
\hline$\omega_{s}, \%$ & Ref. & Comment \\
\hline 0.58 & {$[74]$} & Theory for $\varphi=1.2 \mathrm{LHD}$ \\
\hline 0.58 & {$[75]$} & Theory for $\varphi=1.2 \mathrm{LHD}$ \\
\hline 0.65 & {$[76]$} & Theory for $\varphi=1.2 \mathrm{LHD}$ \\
\hline $0.43 \pm 0.05 \pm 0.06$ & {$[77]$} & LAMPF experiment for $\varphi=1.2 \mathrm{LHD}$ \\
\hline $0.48 \pm 0.02 \pm 0.04$ & {$[78]$} & PSI experiment for $\varphi=1.2 \mathrm{LHD}$ \\
\hline $0.532 \pm 0.030$ & {$[79]$} & RIKEN experiment for $\varphi=1.2 \mathrm{LHD}$ \\
\hline $0.505 \pm 0.029$ & {$[10]$} & PSI experiment for $\varphi=1.45 \mathrm{LHD}$ \\
\hline $0.573 \pm 0.021^{\text {stat }} \pm 0.032^{\text {syst }}$ & & This experiment for $\varphi=1.22 \mathrm{LHD}$ \\
\hline
\end{tabular}

The mean value $\lambda_{Z} C_{Z}=(0.08 \pm 0.03) \mu \mathrm{s}^{-1}$ obtained in fits is in agreement with the estimate $(0.08 \pm 0.04) \mu \mathrm{s}^{-1}$ based on the analysis of the electron time spectra. Product $\lambda_{t t \mu} \omega_{t t}$ being free obtained as $0.28 \pm 0.15$ which agrees with $[70,71,72]$.

Table 5: Results of the fit of the data for gaseous $D / T$ at low temperatures $(45-300 \mathrm{~K}) . a$ is the parameter of formulae (20) for the $q_{1 S}$ approximation

\begin{tabular}{|c|c|c|c|}
\hline Parameter & Value & Conditions & \\
\hline$a$ & $7.1 \pm 2.5 \quad$ free & & \\
\hline \multirow[t]{9}{*}{$\lambda_{d t \mu-d}$} & $326 \pm 27 \quad$ free & $T=45 \mathrm{~K}$ & $\varphi=0.45 \mathrm{LHD}$ \\
\hline & $403 \pm 32$ & $T=45 \mathrm{~K}$ & $\varphi=0.67 \mathrm{LHD}$ \\
\hline & $490 \pm 36$ & $T=45 \mathrm{~K}$ & $\varphi=0.95 \mathrm{LHD}$ \\
\hline & $320 \pm 26$ & $T=158 \mathrm{~K}$ & $\varphi=0.43 \mathrm{LHD}$ \\
\hline & $402 \pm 32$ & $T=158 \mathrm{~K}$ & $\varphi=0.66 \mathrm{LHD}$ \\
\hline & $499 \pm 37$ & $T=158 \mathrm{~K}$ & $\varphi=0.90 \mathrm{LHD}$ \\
\hline & $292 \pm 29$ & $T=300 \mathrm{~K}$ & $\varphi=0.31 \mathrm{LHD}$ \\
\hline & $313 \pm 30$ & $T=300 \mathrm{~K}$ & $\varphi=0.42 \mathrm{LHD}$ \\
\hline & $380 \pm 33$ & $T=300 \mathrm{~K}$ & $\varphi=0.52 \mathrm{LHD}$ \\
\hline$\lambda_{d t \mu-t}$ & $\begin{array}{l}7 \pm 4 \\
52 \pm 14\end{array}$ & $\begin{array}{l}T=45-160 \mathrm{~K} \\
T=300 \mathrm{~K}\end{array}$ & $\begin{array}{l}\varphi=0.4-1.0 \text { LHD } \\
\varphi=0.3-0.5 \text { LHD }\end{array}$ \\
\hline
\end{tabular}


5.2.2. Low temperature gaseous $D / T$ data. Here we present new data, related to the mixture temperature $T=45,158 \mathrm{~K}$ and different densities $\varphi=(0.2-$ 1) LHD. First of all, it is interesting from the point of view of the density dependence of the $d t \mu$-molecule formation rate on $D_{2}$ molecules.

For all values of $\varphi$ approximation (20) for $q_{1 S}$ was used in the fit. The results are presented in Table 5. The data for $T=300 \mathrm{~K}$ recently presented in [26] is also included. As can see, the obtained data for $\lambda_{d t \mu-d}$ demonstrate the strong density dependence and practically do not sensitive to the mixture temperature.

5.2.3. High temperature gaseous $D / T$ data. Measurements for high temperatures $(T \geq 300 \mathrm{~K})$ are important for the determination of the $d t \mu$-molecule formation rate on $D T$ molecules. According to the standard theory, in this region the Maxwell distribution for the $t \mu$ energy $\left(E_{t \mu}\right)$ overlaps the nearest resonance $\lambda_{d t \mu-t}\left(E_{t \mu}\right)$. Besides, it is interesting to clarify for which temperatures $\lambda_{d t \mu-d}$ keeps to be density depended and which is its temperature dependence.

The preliminary data for $T=300,550,800 \mathrm{~K}$ were presented in [26]. Now we present the final data obtained from the common fit of the experimental dependencies $\lambda_{c}(\varphi, T)$. The $d t \mu$-molecule formation rates on $D_{2}$ and $D T$ molecules were put independent on density at temperatures $T>300 \mathrm{~K}$. They are presented in Table 6.

Table 6: Results of the fit of the data for gaseous D/T at high temperatures (300-800 K)

\begin{tabular}{llll|ll}
\hline \multicolumn{1}{l|}{ Parameter } & Value & & Conditions & \\
\hline$a$ & & $8.5 \pm 2.8$ & free & & \\
\hline$\lambda_{d t \mu-t}$ & $\mu \mathrm{s}^{-1}$ & $56 \pm 14$ & free & $T=300 \mathrm{~K}$ & $\varphi=0.2-0.8 \mathrm{LHD}$ \\
& $190 \pm 81$ & free & $T=500 \mathrm{~K}$ & $\varphi=0.2-0.8 \mathrm{LHD}$ \\
& & $198 \pm 28$ & free & $T=550 \mathrm{~K}$ & $\varphi=0.2-0.8 \mathrm{LHD}$ \\
& & $270 \pm 53$ & free & $T=635 \mathrm{~K}$ & $\varphi=0.2-0.8 \mathrm{LHD}$ \\
& $328 \pm 34$ & free & $T=800 \mathrm{~K}$ & $\varphi=0.2-0.8 \mathrm{LHD}$ \\
\hline$\lambda_{d t \mu-d}$ & $\mu \mathrm{s}^{-1}$ & $251 \pm 36$ & free & $T=300 \mathrm{~K}$ & $\varphi=0.20 \mathrm{LHD}$ \\
& & $277 \pm 30$ & free & $T=300 \mathrm{~K}$ & $\varphi=0.31 \mathrm{LHD}$ \\
& $293 \pm 31$ & free & $T=300 \mathrm{~K}$ & $\varphi=0.42 \mathrm{LHD}$ \\
& & $354 \pm 34$ & free & $T=300 \mathrm{~K}$ & $\varphi=0.52 \mathrm{LHD}$ \\
& $420 \pm 44$ & free & $T=300 \mathrm{~K}$ & $\varphi=0.78 \mathrm{LHD}$ \\
& & $319 \pm 45$ & free & $T=500-800 \mathrm{~K}$ & $\varphi=0.19-0.60 \mathrm{LHD}$ \\
\hline
\end{tabular}

The following conclusions can be made under their consideration.

1. According to theory, $\lambda_{d t \mu-t}$ rises with temperature.

2. Contrary this, $\lambda_{d t \mu-d}$ does not revel the temperature dependence.

5.2.4. Common fit of gaseous $D / T$ data. To reliably extract $\lambda_{d t \mu}$ for given $T$ and $\varphi$ each set of $\lambda_{c}\left(\varphi, T ; C_{t}\right)$ should contain enough points corresponding to a wide range of $C_{t}$. Not all our data satisfied this condition. We could 

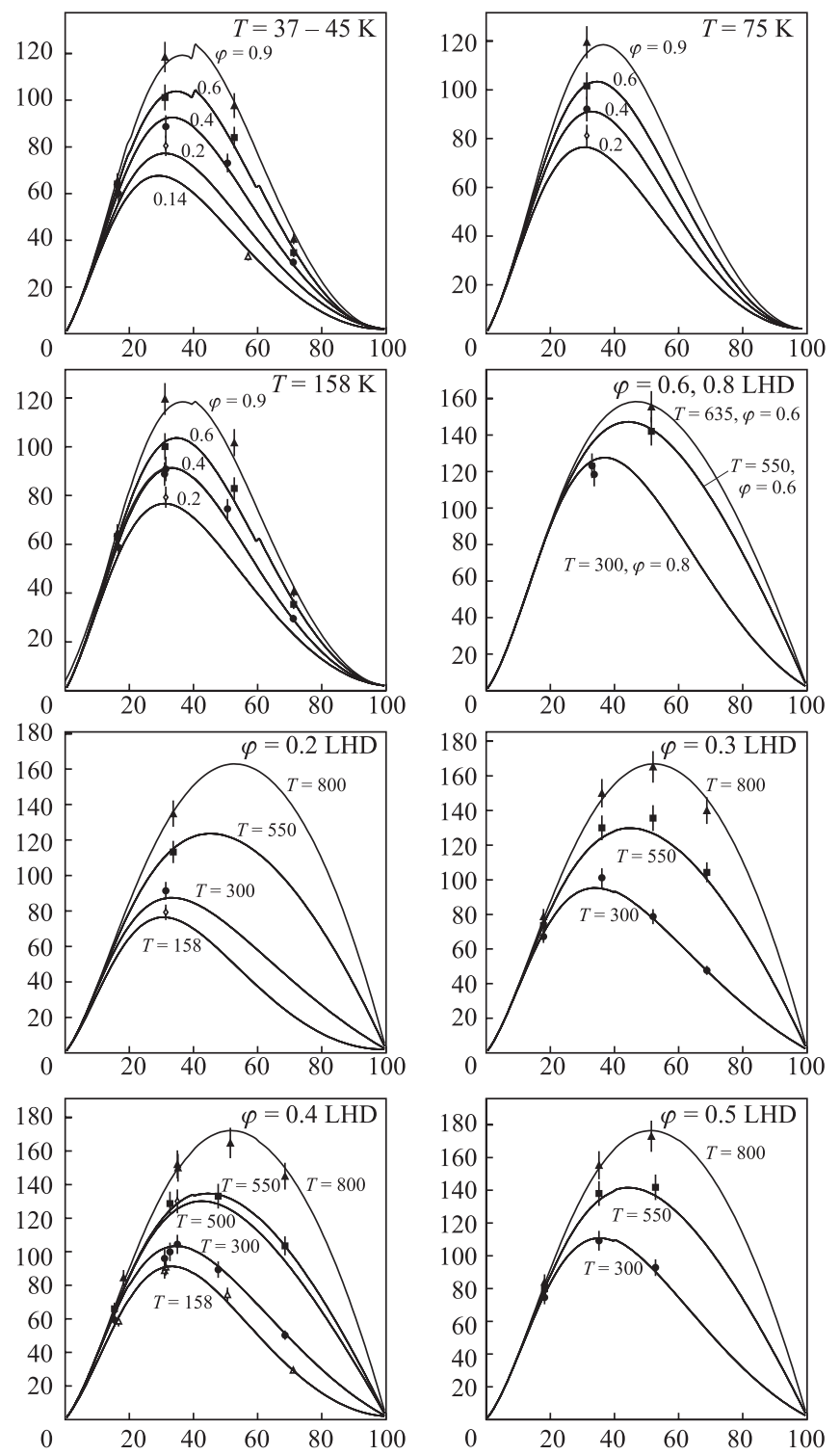

Fig. 14. Common fit of the normalized cycling rates as a function of the tritium concentration for all (76 points) data for the gaseous $D / T$ mixture $(T=37-800 \mathrm{~K}, \varphi=$ 0.143-1.024 LHD). Lines are the optimum fit 

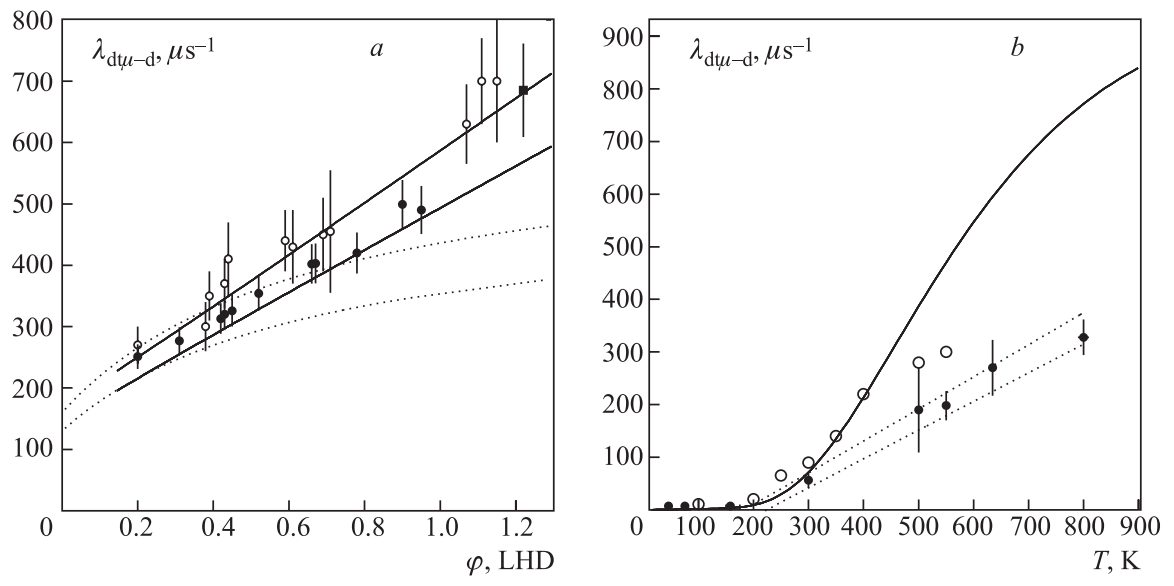

Fig. 15. $a-\lambda_{d t \mu-d}$ as a function of density for $T \leq 300 \mathrm{~K}$. Filled circles are our points for gas, empty circles are the results of LAMPF [17], the square is the result of the present paper for liquid (21). Solid lines are the permissible values found from the fit. Dashed lines are limits for the $\lambda_{d t \mu-d}$ region obtained in [14]. $b-\lambda_{d t \mu-t}$ as a function of temperature. Filled circles are our points, empty circles are the results of LAMPF [17]. The solid line is the theory [13] for $\lambda_{d t \mu-t}^{0}$. Dashed lines are limits of parametrization (25)

not conduct measurements with high $C_{t}$ at «extreme» $\varphi$ and $T$. Nevertheless, we could include all data in the analysis making some assumptions on the $\lambda_{d t \mu}$ density and temperature dependencies.

Based on our preliminary analysis [26, 67] and temperature and density dependencies of $\lambda_{c}$ obtained from the analysis of total data we can conclude that

(i) $\lambda_{d t \mu-d}$ rises linearly with density in a wide temperature region up to $T=300 \mathrm{~K}$;

(ii) at temperatures $T=300-800 \mathrm{~K} \lambda_{d t \mu-t}$ is very close to the linear function of temperature and does not depend on density.

So we chose the simplest linear parametrization for the temperature and density dependencies of the formation rates

$\lambda_{d t \mu-d}(\varphi)=A_{d}+B_{d} \cdot \varphi$ at $T=37 \div 300 \mathrm{~K} ; \lambda_{d t \mu-d}=C_{d}$ at $T>300 \mathrm{~K}$

$$
\lambda_{d t \mu-t}(T)=A_{t}+B_{t} \cdot T \text { at } T \geq 200 \mathrm{~K}, \varphi=0.2 \div 0.9 .
$$

General expression (19) was used for $q_{1 S}$ including the density dependence term.

A total of 76 gaseous points of $\lambda_{c}$ were under fitting by using formula (10). The systematic error due to the neutron detection efficiency $\Delta \epsilon_{n}$ (the same for 


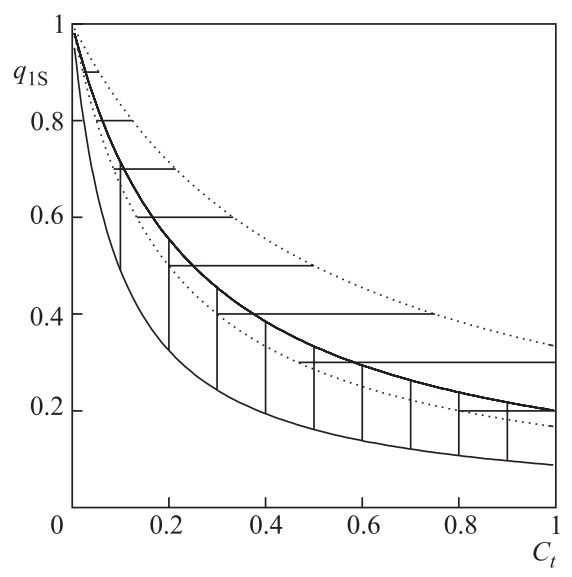

Fig. 16. $q_{1 S}$ as a function of the tritium concentration. The vertical shading is the parametrization obtained by current fit. The horizontal shading is the PSI result [14] based on the measurements at low temperature $T \leq 40 \mathrm{~K}$

all data) was excluded from the errors in the course of fitting. The results are presented in Table 6 and in Figs. 14-16.

From Fig. 14 one can see how the experimental values of $\lambda_{c}$ are described by formulae (10) with our parametrization for $q_{1 S}$ and $\lambda_{d t \mu}$. It follows from the fit that experimental data are in satisfactory agreement with the used approximation: $\chi^{2}=84$ for 76 points and 6 variable parameters. The optimum values of our approximation are presented in Table 7. Note that we obtained that $q_{1 S}$ turned out to be density independent. The same was obtained in [14] but our values of $q_{1 S}$ are somewhat lower than in this work (see Fig. 16).

Table 7. Results of the common fit of the all data for gaseous $D / T$

\begin{tabular}{lll|ll}
\hline Parameter & & & Fit result & \\
\hline$q_{1 S}$ & $b$ & & $7.2 \pm 2.9$ & free \\
& $c$ & & $0 \pm 1$ & free \\
\hline$\lambda_{d t}$ & & $\mu \mathrm{s}^{-1}$ & $280[18,21,35,36]$ & fixed \\
$\lambda_{1-0}$ & & $\mu \mathrm{s}^{-1}$ & $1200[68,69]$ & fixed \\
\hline$\lambda_{d t \mu-d}$ & $A_{d}(T \leq 300 \mathrm{~K})$ & $\mu \mathrm{s}^{-1}$ & $156 \pm 14$ & free \\
& $B_{d}(T \leq 300 \mathrm{~K})$ & $\mathrm{LHD}^{-1}$ & $384 \pm 21$ & free \\
& $C_{d}(T>300 \mathrm{~K})$ & $\mu s^{-1}$ & $331 \pm 32$ & free \\
\hline$\lambda_{d t \mu-t}$ & $T \leq 200 \mathrm{~K}$ & $\mu \mathrm{s}^{-1}$ & $6 \pm 6$ & free \\
& $A_{t}(T \geq 200 \mathrm{~K})$ & $\mu \mathrm{s}^{-1}$ & $-117 \pm 9$ & free \\
& $B_{t}(T \geq 200 \mathrm{~K})$ & $\mathrm{K}^{-1}$ & $0.577 \pm 0.028$ & free \\
\hline
\end{tabular}


Figure 15 represents the fit results for the low temperature $(T \leq 300 \mathrm{~K})$ data for $\lambda_{d t \mu-d}(\varphi)(a)$ and $\lambda_{d t \mu-t}(T)(b)$. The area limited by the straight lines means permissible values found from the fit with taking into account the uncertainties in density and temperature. The obtained fit accuracy turned out to be

$$
\begin{gathered}
\delta\left(\lambda_{d t \mu-d}\right)=(8 \div 9) \% \\
\delta\left(\lambda_{d t \mu-d}\right)=20 \%(T=300 \mathrm{~K}) . \div 9 \%(T=800 \mathrm{~K}) .
\end{gathered}
$$

The systematic error $\delta_{\text {syst }}=7 \%$ should be taken into account under comparison of our data with theory and other measurements.

\section{DISCUSSION}

6.1. Muon Sticking Probability. As can be seen from Table 4, the experimental values of $\omega_{s}$ obtained by different experimental groups are in satisfactory agreement with each other. The value obtained by the direct method remarkably coincides with the one determined from the analysis of the muon losses as a function of the tritium concentration. At the same time there remains some disagreement between experiment and theory.

6.2. $\boldsymbol{q}_{1 S}$ and $\boldsymbol{\lambda}_{\boldsymbol{d} t \boldsymbol{\mu}}$. Analysis of the experimental data confirms the theoretical conclusion about the significant role of the muon transfer from the excited $d \mu$-atom states. According to the theory, the intensity of this process turns out to depend on the tritium concentration. The probability $q_{1 S}$ of muon reaching the $d \mu$ atomic $1 S$ state is successfully described by rather simple expression (20) with the same parameter $a$ for different $C_{t}$. At the same time, contrary to the theoretical predictions, $q_{1 S}$ does not show noticeable density dependence. These conclusions coincide with those made in the PSI [14].

As follows from the theory, at low temperatures $(T<300 \mathrm{~K})$ the process of the $d t \mu$ formation on $D_{2}$ molecules dominates. Non-trivial density dependence $\lambda_{d t \mu-d}(\varphi)$ evidences in favor of the triple collision mechanism (7). Unfortunately, there is still no qualitative agreement between experiment and theory on the intensity of this process. It seems that the absence of noticeable dependence of $\lambda_{d t \mu-d}$ on temperature is hardly to reconcile with the mechanism of $d t \mu$ formation on the negative resonance.

The experimental data on the dependence $\lambda_{d t \mu-d}(\varphi)$ obtained by different experimental groups are in satisfactory agreement. Note, that the parametrization of this dependence suggested by the PSI group is not appropriate for the entire data set.

According to the theoretical predictions about the resonance positions, the process of the $d t \mu$-molecule formation on $D T$ molecules manifests itself at high temperatures $T \simeq 300 \mathrm{~K}$ and rises with temperature. However, both the present 
results and the LAMPF data on $\lambda_{d t \mu-t}(T)$ turned out to be significantly lower than the calculated ones. This means that the intensity of the appropriate resonances is overestimated by theory. The same conclusion follows from the analysis of the epithermal effects in the $d t \mu$ formation made by the PSI group [80]. At the same time, the TRIUMF group, making the TOF measurements of the MCF $d+t$ reaction yield as a function of the $t \mu$-atom energy [81], concluded that their data are in satisfactory agreement with the theory. One should note, however, that the analysis of [81] is very complicated and can involve some systematic uncertainties. To clarify the situation, it is very important to make steady-state measurements at the highest temperatures $T=1000-2000 \mathrm{~K}$ where Maxwell distribution of the $t \mu$-atom energy overlaps the most intensive resonances.

6.3. Possible Influence of the Epithermal Effects. The parameters obtained are related to the steady-state regime when the $t \mu$ atoms formed with the initial energy $E \geq 1 \mathrm{eV}$ already passed through the resonances and are thermalized. However, each time after muon regeneration in the fusion reaction the $\mu$ atoms go through the deceleration stage again feeling the effect of the resonances. Obviously, this leads to an increase in the cycling rate as compared to values related to the Maxwell distributed $\mu$ atoms. This is similar to the well-studied $\mu$ catalysis in low-temperature deuterium related to the two $d \mu$-atom spin states [82]. As in that case, there are «upper» and «lower» states with sharply different cycling rates and quick degradation of the «upper» state. By analogy, one can express the steady-state cycling rate $\lambda_{c}^{s s}$ as

$$
\lambda_{c}^{s s}=\lambda_{c} \cdot(1+\delta),
$$

where $\lambda_{c}$ is the «bare» value.

A relative increase in the cycling rate $\delta$ can be estimated as

$$
\delta \simeq \alpha_{e p} \cdot \lambda_{d t \mu}^{\mathrm{res}} / \lambda_{d}
$$

where $\alpha_{e p}$ is the fraction of the $t \mu$ atoms passing through the resonances during thermalization, $\lambda_{d t \mu}^{\text {res }}$ is the effective $d t \mu$-molecule formation rate in the resonance region and $\lambda_{d}$ is the rate with which $t \mu$ atoms leave the resonance (thermalization and back decay after the $d t \mu$ formation).

Estimations made from the calculated values of $\lambda_{d t \mu}\left(E_{t \mu}\right)$ [13] and the scattering cross-sections $\sigma_{t \mu+d, t \mu+t}$ [83] evidence that the corrections to the steadystate can be as large as tenths of percent. One can expect that the correction $\delta$ should be the smallest for the low tritium concentration because the deceleration rate in $t \mu+d$ collisions is significantly larger than in $t \mu+t$ collisions dominating at high $C_{t}$.

As a consequence, there arises a problem of correctly extracting the $d t \mu$-molecule formation rate from the dependence $\lambda_{c}\left(C_{t}\right)$ given by formulae (10). 
The obvious conclusion is overestimation of $\lambda_{d t \mu}$ as compared with the thermalized $t \mu$-atom situation. In addition, the systematic errors in the parameters of (10) can take place. Fortunately, as is seen from Figs. 13, 14, there is satisfactory agreement between the experimental values of $\lambda_{c}$ and expression (10). Thus, it is believed that the corresponding distortions are not so large. According to our estimations, the appropriate corrections to $\lambda_{d t \mu}$ in the region $C_{t}=20-70 \%$ are $\delta \simeq(10-20) \%$.

\section{CONCLUSION}

The systematic experimental investigations of the MCF process in $D / T$ mixture have been conducted at the JINR Phasotron by the novel method. Measurements were made in a wide range of the mixture parameters - density, temperature and tritium concentration. The variety of the experimental conditions can be seen in Fig. 17, showing the cycling rate vs mixture conditions.
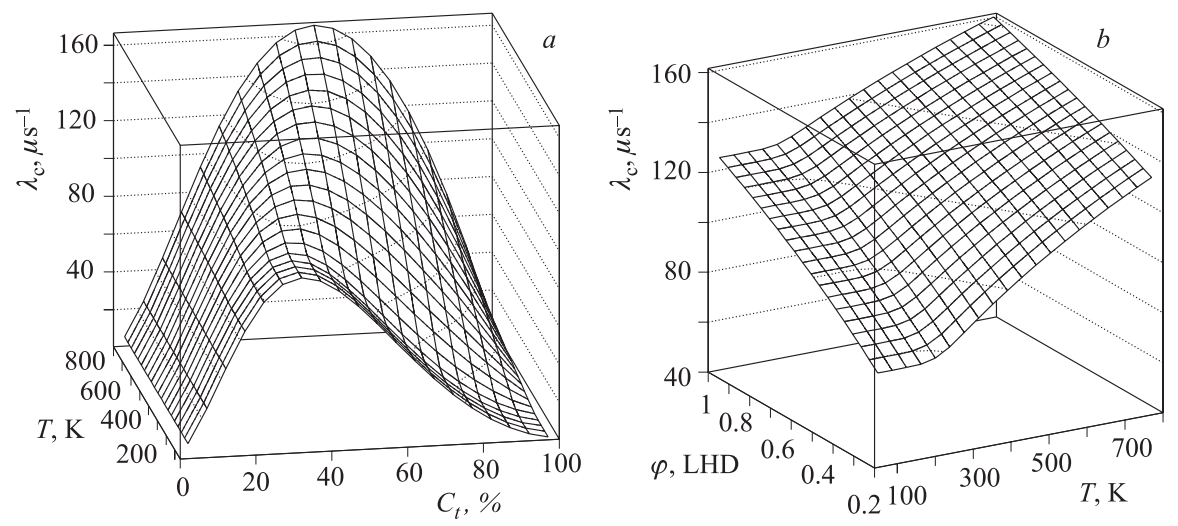

Fig. 17. Normalized cycling rate dependencies on the $D / T$ mixture conditions plotted with the use of optimum parametrizations obtained from fit. $a-$ normalized cycling rate as a function of tritium concentration and temperature for $\varphi=0.4$ LHD. $b-$ normalized cycling rate as a function of temperature and density for $C_{t}=0.35$. Temperature region of $160-300 \mathrm{~K}$ is smoothed

Analysis of the data allows us to determine the basic MCF parameters. In general they are in agreement with the ones obtained by other groups in the region where the experimental conditions were similar. The comparison of the experimental data with the theory confirms the efficiency of the main mechanisms considered in the MCF theory but the full qualitative description of the process is not achieved yet. 
In our opinion, it will be very important to make measurements with a $D / T$ mixture at the highest temperatures $T=(1000-2000) \mathrm{K}$ where the main resonances manifest themselves most effectively.

Acknowledgments. The authors are grateful to Prof. L.I. Ponomarev for the stimulating discussions and D. V. Balin, G. G. Semenchuk, Yu. A. Smirenin, N. I. Voropaev (PNPI, Gatchina) taken part in our first measurements. The work was supported by the ISTC (Project 025-95), the RF Ministry of Atomic Energy (Contract No 6.25.19.19.99.969), the RF Ministry of Science and Technology (State Contract No 103-7(00)-II) and the RFBR (Projects 97-02-16882, 98-02-16351, 00-02-17192, 03-02-16876).

The authors dedicate the article to the memory of Prof. V.P. Dzhelepov their co-author and supervisor. Prof. V.P. Dzhelepov was the initiator of the long-term experimental research on Muon Catalyzed Fusion (MCF) and till his last days was at the head of this scientific branch at JINR.

\section{REFERENCES}

1. Ponomarev L. I. // Contemp. Phys. 1990. V.31. P. 219.

2. Breunlich W. H., Kammel P., Cohen J. S., Leon M. // Ann. Rev. Nucl. Sci. 1998. V. 24. P. 151.

3. Gerstein S. S., Petrov Yu. V., Ponomarev L. I. // Usp. Fiz. Nauk. 1990. V. 160. P. 3.

4. Ponomarev L. I. // Hyp. Interact. 2001. V. 138. P. 15.

5. Ponomarev L. I. // Proc. of the Intern. Workshop on Exotic Atoms, 28-30 November 2002. Vienna, Austria. Austrian Academy of Science Press, 2003.

6. Filchenkov V. V., Grafov N. N. JINR Commun. E15-2003-96. Dubna, 2003.

7. Bogdanova L. N., Filchenkov V. V. // Hyperfine Inter. 2001. V.138. P. 321.

8. Petitjean C. et al. // Fusion Technology. 1994. V.25. P.466; Petrov Yu. V., Sakhnovsky E. G. // Hyperfine Inter. 1996. V. 101/102. P. 647; Anisimov V. et al. // Fusion Technology. 2001. V. 39. P. 198.

9. Petrov Yu. V. // Nature (London). 1980. V.285. P. 466.

10. Petitjean C. // Hyp. Interact. 2001. V. 138. P. 191.

11. Voropaev N. I. et al. // Hyp. Interact. 2001. V.138. P. 331.

12. Faifman M. P., Menshikov L. I., Strizh T.A. // Muon Cat. Fusion. 1989. V.4. P. 1.

13. Faifman M. P. et al. // Hyp. Interact. 1996. V. 101/102. P. 179.

14. Ackerbauer P. et al. // Nucl. Phys. A. 1999. V.652. P. 311.

15. Ackerbauer P. et al. // Hyp. Interact. 1993. V. 82. P. 357.

16. Kawamura N. et al. // Hyp. Interact. 2001. V. 138. P. 235.

17. Jones S. E. et al. // Phys. Rev. Lett. 1983. V.51. P.1757; Jones S. E. et al. // Phys. Rev. Lett. 1986. V.56. P. 588.

18. Jones S. E. et al. // Muon Cat. Fusion. 1987. V.1. P.21; Caffrey A.J. et al. // Muon Cat. Fusion. 1987. V.1. P. 53. 
19. Dzhelepov V. P. et al. // Zh. Exp. Teor. Fiz. 1966. V.50. P.1235. (Sov. Phys. JETP 1966. V.23. P. 820).

20. Bystritsky V.M. et al. // Zh. Exp. Teor. Fiz. 1979. V.76. P. 460.

21. Bystritsky V. M. et al. // Phys. Lett. B. 1980. V.94. P. 476; Zh. Exp. Teor. Fiz. 1981. V. 80. P. 1700; JETP Lett. 1981. V.53. P. 877.

22. Vinitsky S. I. et al. // Zh. Exp. Teor. Fiz. 1978. V.74. P. 849. (Sov. Phys. JETP 1979. V. 47. P. 444).

23. Averin Yu.P. et al. // Hyp. Interact. 1998. V.118. P. 111.

24. Bom V.R. et al. // Hyp. Interact. 1998. V.118. P. 103.

25. Averin Yu. P. et al. // Hyp. Interact. 1998. V. 118. P. 121.

26. Bom V.R. et al. // Hyp. Interact. 2001. V. 138. P. 213; Demin D. L. et al. // JINR Commun. E15-2000-157. Dubna, 2000.

27. Menshikov L.I., Ponomarev L.I. // Pis'ma Zh. Exp. Teor. Fiz. 1984. V.39. P. 542. [JETP Lett. 1984. V.39. P. 663].

28. Menshikov L.I., Ponomarev L.I. // Pis'ma Zh. Exp. Teor. Fiz. 1985. V.42. P. 12. [JETP Lett. 1985. V.42. P. 13].

29. Menshikov L. I., Ponomarev L. I. // Z. Phys. D. 1986. V.2. P. 1.

30. Markushin V.E. // Phys. Rev. A. 1994. V.50. P. 1137.

31. Czaplinski W. et al. // Phys. Rev. A. 1994. V.50. P.525; Phys. Rev. A. 1994. V. 50. P. 518.

32. Faifman M. P., Menshikov L. I. // Hyperfine Inter. 2001. V. 138. P. 61.

33. Markushin V.E., Jensen T. S. // Hyperfine Inter. 2001. V. 138. P. 71.

34. Kravtsov A. V. et al. // Hyperfine Inter. 2001. V. 138. P. 103.

35. Breunlich W. H. et al. // Phys. Rev. Lett. 1987. V.58. P. 329.

36. Balin D. V. et al. // Zh. Eksp. Teor. Fiz. 1987. V.92. P. 1543. (Sov. Phys. JETP 1987. V.65. P. 866).

37. Vesman E. // Pis'ma Zh. Eksp. Teor. Fiz. 1967. V.5. P.113. (Sov. Phys. JETP Lett. 1967. V.5. P.91).

38. Bogdanova L. N. et al. // Zh. Exp. Teor. Fiz. 1982. V. 83. P. 1615.

39. Menshikov L. I., Ponomarev L. I. // Phys. Lett. B. 1986. V. 167. P. 141.

40. Leon M. // Muon Cat. Fusion. 1987. V. 1. P. 163; Phys. Rev. A. 1994. V. 49. P. 4438.

41. Petrov Yu. V., Petrov V. Yu., Schmidt H. H. // Phys. Lett. B. 1994. V. 331. P. 266.

42. Demianov A. V. et al. JINR Commun. P9-93-374. Dubna, 1993.

43. Demin D. L. et al. // Hyp. Interact. 1999. V. 119. P. 349; Prib. Tekhn. Exp. 1999. V. 1. P. 21; JINR Preprint P13-97-243. Dubna, 1997.

44. Perevozchikov V. V. et al. // Hyp. Interact. 1998. V.119. P.353; Prib. Tekhn. Exp. 1999. V. 1. P. 28; JINR Preprint D15-98-107. Dubna, 1998.

45. Perevozchikov $V$. $V$. et al. High-pressure targets to study muon catalyzed nuclear fussion. // Proc. of the Second International Workshop Interaction of Hydrogen Isotopes with Structural Materials. IHISM-04, Sarov, April 12-17, 2004. Fusion Science and Technology (in press).

46. Yukhimchuk A. A. et al. // Hyp. Interact. 1998. V. 119. P. 341.

47. Konin A. D. JINR Commun. P13-82-634. Dubna, 1982. 
48. Dzhelepov V. P. et al // Nucl. Instr. Meth. A. 1988. V.269. P. 634;

Filchenkov V. V., Konin A. D., Zinov V. G. // Nucl. Instr. Meth. A. 1986. V. 245. P. 490; Baranov V. A. et al. // Nucl. Instr. Meth. A. 1996. V.374. P.335;

Zinov V. G. et al. JINR Commun. P13-91-182. Dubna, 1991.

49. Filchenkov V. V., Konin A.D., Rudenko A.I. // Nucl. Instr. Meth. A. 1990. V. 294. P. 504.

50. Zinov V. G. et al. // Prib. Techn. Exp. 1998. V.3. P. 38.

51. Malkov M. P. et al. Guide to Physico-Technical Base of Cryogenic. M.: Energoatomizdat, 1973.

52. Prydz R., Timmerhaus K.D., Stewart R. B. // Advances in Cryogenic Engineering. 1967. V. 13. P. 384.

53. Yukhimchuk A. A. et al. Preprint VNIIEF 83-2002. Sarov, 2002.

54. Demin D. L. et al. // Journ. of Low Temp. Phys. 2000. V.120. P.45; JINR Preprint P8-99-179. Dubna, 1999.

55. Demin D.L., Grafov N.N. Determination of Concentrations and Nuclear Density of Hydrogen Isotope Mixtures in MCF Experiments with Liquid Tritium Target. Report of LNP, JINR, 1997.

56. Schellenberg L. // Muon Cat. Fusion. 1990/91. V.5/6. P.73; Hyp. Interact. 1993. V.82. P. 513.

57. Gartner B. et al. // Hyp. Interact. 1999. V. 119. P. 103.

58. Kawamura N. et al. // Hyp. Interact. 1999. V.118. P. 213; Kawamura N. et al. // Phys. Lett. B. 1999. V.465. P.74.

59. Filchenkov V. V., Drebushko A. E., Rudenko A. I. // Nucl. Instr. Meth. A. 1997. V. 395. P. 237.

60. Zinov V. G. // Muon Cat. Fusion 1992. V.7. P. 419.

61. Filchenkov V. V. // Muon Cat. Fusion 1992. V.7. P. 409.

62. Filchenkov V. V., Sadetsky S. M. // Nucl. Instr. Meth. A. 2002. V.480. P.771; JINR Preprint E15-2000-223. Dubna, 2000.

63. Filchenkov V. V. et al. JINR Preprint E15-2002-285. Dubna, 2002; Hyp. Interact., 2004 (in press).

64. Bom V.R., Filchenkov V. V. // Hyp. Interact. 1999. V.119. P.365; JINR Preprint E15-98-338. Dubna, 1998;

Filchenkov V. V., Marczis L. JINR Commun. E13-88-566. Dubna, 1988.

65. Filchenkov V. V. JINR Commun. E15-2000-224. Dubna, 2000.

66. Jensen T. S., Markushin V. E. // Eur. Phys. J. D. 2002. V.21. P. 271.

67. Filchenkov V. V., Grafov N. N. // Hyp. Interact. 2001. V. 138. P. 241.

68. Bracci L. et al. // Phys. Lett. A. 1989. V.134. P. 435; Muon Cat. Fusion. 1989. V. 4. P. 247.

69. Abramov D. I., Gusev V. V., Ponomarev L. I. // Hyp. Interact. 2001. V. 138. P. 275.

70. Breunlich W. H. et al. // Phys. Rev. Lett. 1984. V.53, No.12. P. 1137;

Breinlich W. H. et al. // Muon Cat. Fusion. 1987. V. 1. P. 29;

Muon Cat. Fusion 1987. V.1. P. 67; Muon Cat. Fusion. 1987. V.1. P. 121.

71. Matsuzaki T. et al. // Hyp. Interact. 1999. V.118. P.229; Phys. Lett. B. 2003. V. 557. P. 176. 
72. Bogdanova L. N. // Muon Cat. Fusion. 1988. V.3. P. 359.

73. Ishida K. et al. // Phys. Rev. Lett. 2003. V.90. P.043401;

Ishida K. et al. // Hyp. Interact. 1999. V. 118. P. 203.

74. Struensee M., Cohen J. S. // Phys. Rev. A. 1988. V.38. P. 44.

75. Markushin V. E. // Muon Cat. Fusion. 1988. V.3. P. 395.

76. Kamimura M. et al. // Hyp. Interact. 1999. V.118. P. 217.

77. Jones S. E., Taylor S. F., Anderson A. N. // Hyp. Interact. 1993. V.82. P. 03.

78. Petitjean C. // Hyp. Interact. 1993. V. 82. P. 273.

79. Ishida K. et al. // Hyp. Interact. 2001. V. 138. P. 225.

80. Jeitler M. et al. // Phys. Rev. A. 1995. V.51. P. 2881.

81. Fujiwara M. C. et al. // Phys. Rev. Lett. 2000. V. 85. P. 1642.

82. Filchenkov V. V. // Hyp. Interact. 1996. V. 101/102. P. 37.

83. Adamczak A. et al. // Atomic Data and Nuclear Data Tables. 1996. V. 62. P. 255; JINR Preprint E4-95-488. Dubna, 1995.

Received on August 20, 2004. 


\section{Корректор T. Е. Попеко}

Подписано в печать 24.09.2004.

Формат $60 \times 90 / 16$. Бумага офсетная. Печать офсетная.

Усл. печ. л. 2,68. Уч.-изд. л. 3,78. Тираж 330 экз. Заказ № 54611.

Издательский отдел Объединенного института ядерных исследований 141980, г. Дубна, Московская обл., ул. Жолио-Кюри, 6.

E-mail: publish@pds.jinr.ru www.jinr.ru/publish/ 